\title{
Multi-level direct K-way hypergraph partitioning with multiple constraints and fixed vertices ${ }^{\text {th }}$
}

\author{
Cevdet Aykanat ${ }^{\mathrm{a}, *}$, B. Barla Cambazoglu ${ }^{\mathrm{b}}$, Bora Uçar ${ }^{\mathrm{c}, 1}$ \\ ${ }^{\mathrm{a}}$ Computer Engineering Department, Bilkent University, 06800 Bilkent, Ankara, Turkey \\ ${ }^{\mathrm{b}}$ Department of Biomedical Informatics, Ohio State University, Columbus, OH 43210, USA \\ ${ }^{\mathrm{c}}$ CERFACS, 42. Av. G. Coriolis, 31057 Toulouse, France
}

Received 27 July 2006; received in revised form 20 September 2007; accepted 25 September 2007

Available online 7 October 2007

\begin{abstract}
$K$-way hypergraph partitioning has an ever-growing use in parallelization of scientific computing applications. We claim that hypergraph partitioning with multiple constraints and fixed vertices should be implemented using direct $K$-way refinement, instead of the widely adopted recursive bisection paradigm. Our arguments are based on the fact that recursive-bisection-based partitioning algorithms perform considerably worse when used in the multiple constraint and fixed vertex formulations. We discuss possible reasons for this performance degradation. We describe a careful implementation of a multi-level direct $K$-way hypergraph partitioning algorithm, which performs better than a well-known recursive-bisection-based partitioning algorithm in hypergraph partitioning with multiple constraints and fixed vertices. We also experimentally show that the proposed algorithm is effective in standard hypergraph partitioning.
\end{abstract}

(C) 2007 Elsevier Inc. All rights reserved.

Keywords: Hypergraph partitioning; Multi-level paradigm; Recursive bisection; Direct $K$-way refinement; Multi-constraint; Fixed vertices

\section{Introduction}

\subsection{Motivation}

In the literature, combinatorial models based on hypergraph partitioning are proposed for various complex and irregular problems arising in parallel scientific computing $[4,10,17,26,50,53]$, VLSI design $[2,42]$, software engineering [6], and database design $[22,23,41,43,46]$. These models formulate an original problem as a hypergraph partitioning problem, trying to optimize a certain objective function (e.g., minimizing the total volume of communication in parallel

\footnotetext{
This work is partially supported by the Scientific and Technological Research Council of Turkey (TÜBITAK) under project EEEAG-106E069.

* Corresponding author. Fax: +903122664047.

E-mail addresses: aykanat@cs.bilkent.edu.tr (C. Aykanat), barla@bmi.osu.edu (B.B. Cambazoglu), ubora@cerfacs.fr (B. Uçar).

${ }^{1}$ The work of this author was supported by the Scientific and Technological Research Council of Turkey (TÜBITAK) under the program 2219, by the University Research Committee of Emory University, and by Agence Nationale de la Recherche through project ANR-06-CIS6-010.
}

volume rendering, optimizing the placement of circuitry on a dice area, minimizing the access to disk pages in processing GIS queries) while maintaining a constraint (e.g., balancing the computational load in a parallel system, using disk page capacities as an upper bound in data allocation) imposed by the problem. In general, the solution quality of the hypergraph partitioning problem directly relates to the formulated problem. Hence, efficient and effective hypergraph partitioning algorithms are important for many applications.

\subsection{Definitions}

A hypergraph $\mathcal{H}=(\mathcal{V}, \mathcal{N})$ consists of a set of vertices $\mathcal{V}$ and a set of nets $\mathcal{N}$ [5]. Each net $n_{j} \in \mathcal{N}$ connects a subset of vertices in $\mathcal{V}$. The set of vertices connected by a net $n_{j}$ are called its pins and denoted as $\operatorname{Pins}\left(n_{j}\right)$. The size of a net $n_{j}$ is equal to the number of its pins, that is, $s\left(n_{j}\right)=\left|\operatorname{Pins}\left(n_{j}\right)\right|$. A cost $c\left(n_{j}\right)$ is associated with each net $n_{j}$. The nets connecting a vertex $v_{i}$ are called its nets and denoted as $\operatorname{Nets}\left(v_{i}\right)$. The degree of a vertex $v_{i}$ is equal to the number of its nets, that is, $d\left(v_{i}\right)=\left|\operatorname{Nets}\left(v_{i}\right)\right|$. A weight $w\left(v_{i}\right)$ is associated with each 
vertex $v_{i}$. In case of multi-constraint partitioning, multiple weights $w^{1}\left(v_{i}\right), w^{2}\left(v_{i}\right), \ldots, w^{T}\left(v_{i}\right)$ may be associated with a vertex $v_{i}$, where $T$ is the number of constraints.

$\Pi=\left\{\mathcal{V}_{1}, \mathcal{V}_{2}, \ldots, \mathcal{V}_{K}\right\}$ is a $K$-way vertex partition if each part $\mathcal{V}_{k}$ is non-empty, parts are pairwise disjoint, and the union of parts gives $\mathcal{V}$. In $\Pi$, a net is said to connect a part if it has at least one pin in that part. The connectivity set $\Lambda_{j}$ of a net $n_{j}$ is the set of parts connected by $n_{j}$. The connectivity $\lambda_{j}=\left|\Lambda_{j}\right|$ of a net $n_{j}$ is equal to the number of parts connected by $n_{j}$. If $\lambda_{j}=1$, then $n_{j}$ is an internal net. If $\lambda_{j}>1$, then $n_{j}$ is an external net and is said to be cut.

The $K$-way hypergraph partitioning problem (e.g., see [2]) is defined as finding a vertex partition $\Pi=\left\{\mathcal{V}_{1}, \mathcal{V}_{2}, \ldots, \mathcal{V}_{K}\right\}$ for a given hypergraph $\mathcal{H}=(\mathcal{V}, \mathcal{N})$ such that a partitioning objective defined over the nets is optimized while a partitioning constraint is maintained.

In general, the partitioning objective is to minimize a cost function defined over the cut nets. Frequently used cost functions [42] include the cut-net metric

$$
\operatorname{cutsize}(\Pi)=\sum_{n_{j} \in \mathcal{N}_{\text {cut }}} c\left(n_{j}\right),
$$

where each cut net incurs its cost to cutsize $(\Pi)$, and the connectivity-1 metric

$$
\operatorname{cutsize}(\Pi)=\sum_{n_{j} \in \mathcal{N}_{\text {cut }}} c\left(n_{j}\right)\left(\lambda_{j}-1\right),
$$

where each cut net $n_{j}$ incurs a cost of $c\left(n_{j}\right)\left(\lambda_{j}-1\right)$ to cutsize( $\Pi)$. In Eqs. (1) and (2), $\mathcal{N}_{\text {cut }}$ denotes the set of cut nets. In this work, we use the connectivity-1 metric.

Typically, the partitioning constraint is to maintain one or more balance constraints on the part weights. A partition $\Pi$ is said to be balanced if each part $\mathcal{V}_{k}$ satisfies the balance criteria

$$
\begin{gathered}
W^{t}\left(\mathcal{V}_{k}\right) \leqslant\left(1+\varepsilon^{t}\right) W_{\mathrm{avg}}^{t} \quad \text { for } k=1,2, \ldots, K \\
\text { and } t=1,2, \ldots, T .
\end{gathered}
$$

In Eq. (3), for the $t$ th constraint, each weight $W^{t}\left(\mathcal{V}_{k}\right)$ of a part $\mathcal{V}_{k}$ is defined as the sum of the weights $w^{t}\left(v_{i}\right)$ of the vertices in that part, $W_{\text {avg }}^{t}$ is the weight that each part must have in the case of perfect balance, and $\varepsilon^{t}$ is the maximum imbalance ratio allowed. In case of hypergraph partitioning with fixed vertices [1], there is an additional constraint on part assignment of some vertices, i.e., a number of vertices are assigned to parts prior to partitioning with the condition that, at the end of the partitioning, those vertices will remain in the part that they are assigned to.

\subsection{Issues in hypergraph partitioning}

The hypergraph partitioning problem is known to be NP-hard [42], and the algorithms used in partitioning a hypergraph are heuristics. Consequently, the partitioning algorithms must be carefully designed and implemented for increasing the quality of the optimization. At the same time, the computational overhead due to the partitioning process should be minimized in case this overhead is a part of the entire cost to be minimized (e.g., the duration of preprocessing within the total run-time of a parallel application).

The very first works (mostly in the VLSI domain) on hypergraph partitioning used the recursive bisection (RB) paradigm. In the RB paradigm, a hypergraph is recursively bisected (i.e., two-way partitioned) until the desired number of parts is obtained. At each bisection step, cut-net removal and cut-net splitting techniques [16] are adopted to optimize the cut-net and connectivity-1 metrics, respectively. Iterative improvement heuristics based on vertex moves or swaps between the parts are used to refine bisections to decrease the cutsize. The performance of iterative improvement heuristics deteriorate in partitioning hypergraphs with large net sizes [36] and small vertex degrees [29]. Moreover, those improvement heuristics do not have a global view of the problem, and hence solutions are usually far from being optimal.

The multi-level hypergraph partitioning approach emerged as a remedy to these problems [8]. In multi-level bisection, the original hypergraph is coarsened into a smaller hypergraph after a series of coarsening levels, in which highly coherent vertices are grouped into supervertices, thus decreasing the sizes of the nets. After the bisection of the coarsest hypergraph, the generated coarse hypergraphs are uncoarsened back to the original, flat hypergraph. At each uncoarsening level, a refinement heuristic (e.g., FM [28] or KL [39]) is applied to minimize the cutsize while maintaining the partitioning constraint. The multilevel partitioning approach has proven to be very successful $[16,30,33,34,36]$ in optimizing various objective functions.

With the widespread use of hypergraph partitioning in modeling computational problems outside the VLSI domain, the RB scheme adopting the FM-based local improvement heuristics turned out to be inadequate due to the following reasons. First, in partitioning hypergraphs with large net sizes, if the partitioning objective depends on the connectivity of the nets (e.g., the connectivity-1 metric), good partitions cannot always be obtained. The possibility of finding vertex moves that will reduce the cutsize is limited, especially at the initial bisection steps, where net sizes are still large, as nets with large sizes are likely to have large numbers of pins on both parts of the bisection [36]. Second, in partitioning hypergraphs with large variation in vertex weights, targeted balance values may not always be achieved since the imbalance ratio needs to be adaptively adjusted at each bisection step. Third, the RB scheme's nature of partitioning hypergraphs into two equally weighted parts restricts the solution space. In general, imbalanced bisections have the potential to lead to better cutsizes [47]. Finally, several formulations that are variations of the standard hypergraph partitioning problem (e.g., multiple balance constraints, multi-objective functions, fixed vertices), which have recently started to find application in the literature, are not appropriate for the RB paradigm.

As stated above, the RB scheme performs rather poorly in problems where a hypergraph representing the computational structure of a problem is augmented by imposing more than 
one constraints on vertex weights or introducing a set of fixed vertices into the hypergraph. In the multi-constraint partitioning case, the solution space is usually restricted since multiple constraints may further restrict the movement of vertices between the parts. Equally weighted bisections have a tendency to minimize the maximum of the imbalance ratios (according to multiple weights) to enable the feasibility of the following bisections. This additional restriction has manifested itself as $20-30 \%$ degradation in cutsizes with respect to the singleconstraint formulation in some recent applications [35,51,54].

In partitioning with fixed vertices, the RB-based approaches use fixed vertices to guide the partitioning at each bisection step. A set of vertices fixed to a certain subset of parts (a practical option is to select the vertices fixed to the first $K / 2$ parts) are placed in the same part in the first bisection step. As there is no other evident placement information, the same kind of action is taken in the following bisection steps as well. Note that this is a restriction since any bisection that keeps the vertices fixed to half of the parts in the same side of the partition is feasible. That is, parts can be relabeled after the partitioning took place according to the fixed vertex information. In other words, there are combinatorially many part labelings that are consistent with the given fixed vertex information, and the RBbased approaches do not explore these labelings during partitioning. Combined with the aforementioned shortcomings of the RB scheme, this can have a dramatic impact on the solution quality. In Section 5.4, we report cutsize improvements up to $33.30 \%$ by a carefully chosen part labeling combined with $K$-way refinement.

\subsection{Contributions}

In this work, we propose a new multi-level hypergraph partitioning algorithm with direct $K$-way refinement. Based on this algorithm, we develop a hypergraph partitioning tool capable of partitioning hypergraphs with multiple constraints. Moreover, we extend the proposed algorithm and the tool in order to partition the hypergraphs with fixed vertices. The extension is to temporarily remove the fixed vertices, partition the remaining vertices, and then optimally assign the fixed vertices to the obtained parts prior to direct $K$-way refinement. The fixed-vertexto-part assignment problem is formulated as an instance of the maximum-weighted bipartite graph matching problem.

We conduct experiments on a wide range of hypergraphs with different properties (i.e., number of vertices, average net sizes). The experimental results indicate that, in terms of both execution time and solution quality, the proposed algorithm performs better than the state-of-the-art RB-based algorithms provided in $\mathrm{PaToH}$ [16]. In the case of multiple constraints and fixed vertices, the obtained results are even superior.

The rest of the paper is organized as follows. In Section 2 , we give an overview of the previously developed hypergraph partitioning tools and a number of problems that are modeled as a hypergraph partitioning problem in the literature. The proposed hypergraph partitioning algorithm is presented in Section 3. In Section 4, we present an extension to this algorithm in order to encapsulate hypergraph partitioning with fixed vertices. In Section 5, we verify the validity of the proposed work by experimenting on well-known benchmark data sets. The paper is concluded in Section 6.

\section{Previous work on hypergraph partitioning}

\subsection{Hypergraph partitioning tools}

Although hypergraph partitioning is widely used in both academia and industry, the number of publicly available tools is limited. Other than the Mondriaan partitioning tool [53], which is specialized on sparse matrix partitioning, there are five general-purpose hypergraph partitioning tools that we are aware of: hMETIS [33], PaToH [16], MLPart [9], Parkway [48], and Zoltan [24], listed in chronological order.

hMETIS [33] is the earliest hypergraph partitioning tool, published in 1998 by Karypis and Kumar. It contains algorithms for both RB-based and direct $K$-way partitioning. The objective functions that can be optimized using this tool are the cut-net metric and the sum of external degrees metric, which simply sums the connectivities of the cut nets. The tool has support for partitioning hypergraphs with fixed vertices.

PaToH [16] was published in 1999 by Çatalyürek and Aykanat. It is a multi-level, RB-based partitioning tool with support for multiple constraints and fixed vertices. Built-in objective functions are the cut-net and connectivity-1 cost metrics. A high number of heuristics for coarsening, initial partitioning, and refinement phases are readily available in the tool.

MLPart [9] was published in 2000 by Caldwell et al. This is an open source hypergraph partitioning tool specifically designed for circuit hypergraphs and partitioning-based placement in VLSI layout design. It has support for partitioning with fixed vertices.

Parkway [48] is the first parallel hypergraph partitioning tool, published in 2004 by Trifunovic and Knottenbelt. It is suitable for partitioning large hypergraphs in multi-processor systems. The tool supports both the cut-net and connectivity- 1 cost metrics.

Also, Sandia National Labs' Zoltan toolkit [25] contains a recently developed parallel hypergraph partitioner [24]. This partitioner is based on the multi-level RB paradigm and currently supports the connectivity-1 cost metric.

\subsection{Applications of hypergraph partitioning}

Hypergraph partitioning has been used in VLSI design $[2,20,32,42,45]$ since 1970 s. The application of hypergraph partitioning in parallel computing starts by the work of Çatalyürek and Aykanat [15,17]. This work addresses 1D (rowwise or columnwise) partitioning of sparse matrices for efficient parallelization of matrix-vector multiplies. Later, Çatalyürek and Aykanat [18,19] and Vastenhouw and Bisseling [53] proposed hypergraph partitioning models for $2 \mathrm{D}$ (non-zero-based) partitioning of sparse matrices. In these models, the partitioning objective is to minimize the total volume of communication while maintaining the computational load balance. These matrix partitioning models are used in different applications that involve repeated matrix-vector multiplies, such as computa- 
tion of response time densities in large Markov models [26], restoration of blurred images [52], and integer factorization in the number field sieve algorithm in cryptology [7].

In parallel computing, there are also hypergraph partitioning models that address objectives other than minimizing the total volume of communication. For example, Aykanat et al. [4] consider minimizing the border size in permuting sparse rectangular matrices into singly bordered block diagonal form for efficient parallelization of linear programming solvers, $\mathrm{LU}$ and QR factorizations. Another example is the communication hypergraph model proposed by Uçar and Aykanat [49] for considering message latency overhead in parallel sparse matrix-vector multiples based on 1D matrix partitioning.

Besides matrix partitioning, hypergraph models are also proposed for other parallel and distributed computing applications. These include workload partitioning in data aggregation [11], image-space-parallel direct volume rendering [10], data declustering for multi-disk databases [41,43], and scheduling file-sharing tasks in heterogeneous master-slave computing environments $[37,38,40]$.

Formulations that extend the standard hypergraph partitioning problem (e.g., multiple vertex weights and fixed vertices) also find application. For instance, multi-constraint hypergraph partitioning is used for 2D checkerboard partitioning of sparse matrices [19] and parallelizing preconditioned iterative methods [51]. Hypergraph partitioning with fixed vertices is used in formulating the remapping problem encountered in imagespace-parallel volume rendering [10].

Finally, we note that hypergraph partitioning also finds application in problems outside the parallel computing domain such as road network clustering for efficient query processing [22,23], pattern-based data clustering [44], reducing software development and maintenance costs [6], topic identification in text databases [13], and processing spatial join operations [46].

\section{3. $K$-way hypergraph partitioning algorithm}

The proposed algorithm follows the traditional multi-level partitioning paradigm. It includes three consecutive phases: multi-level coarsening, initial partitioning, and multi-level $K$-way refinement. Fig. 1 illustrates the algorithm.

\subsection{Multi-level coarsening}

In the coarsening phase, a given flat hypergraph $\mathcal{H}^{0}$ is converted into a sufficiently small hypergraph $\mathcal{H}^{m}$, which has vertices with high degrees and nets with small sizes, after $m$ successive coarsening levels. At each level $\ell$, an intermediate coarse hypergraph $\mathcal{H}^{\ell+1}=\left(\mathcal{V}^{\ell+1}, \mathcal{N}^{\ell+1}\right)$ is generated by coarsening the finer parent hypergraph $\mathcal{H}^{\ell}=\left(\mathcal{V}^{\ell}, \mathcal{N}^{\ell}\right)$. The coarsening phase results in a sequence $\mathcal{H}^{1}, \mathcal{H}^{2}, \ldots, \mathcal{H}^{m}$ of $m$ coarse hypergraphs.

The coarsening at each level $\ell$ is performed by coalescing vertices of $\mathcal{H}^{\ell}$ into supervertices in $\mathcal{H}^{\ell+1}$. For vertex grouping, agglomerative or matching-based heuristics may be used. In our case, we use the randomized heavy-connectivity matching heuristic $[16,17]$. In this heuristic, vertices in vertex set $\mathcal{V}^{\ell}$ are visited in a random order. In the case of unit-cost nets, every visited, unmatched vertex $v_{i} \in \mathcal{V}^{\ell}$ is matched with a currently unmatched vertex $v_{j} \in \mathcal{V}^{\ell}$ that shares the maximum number of

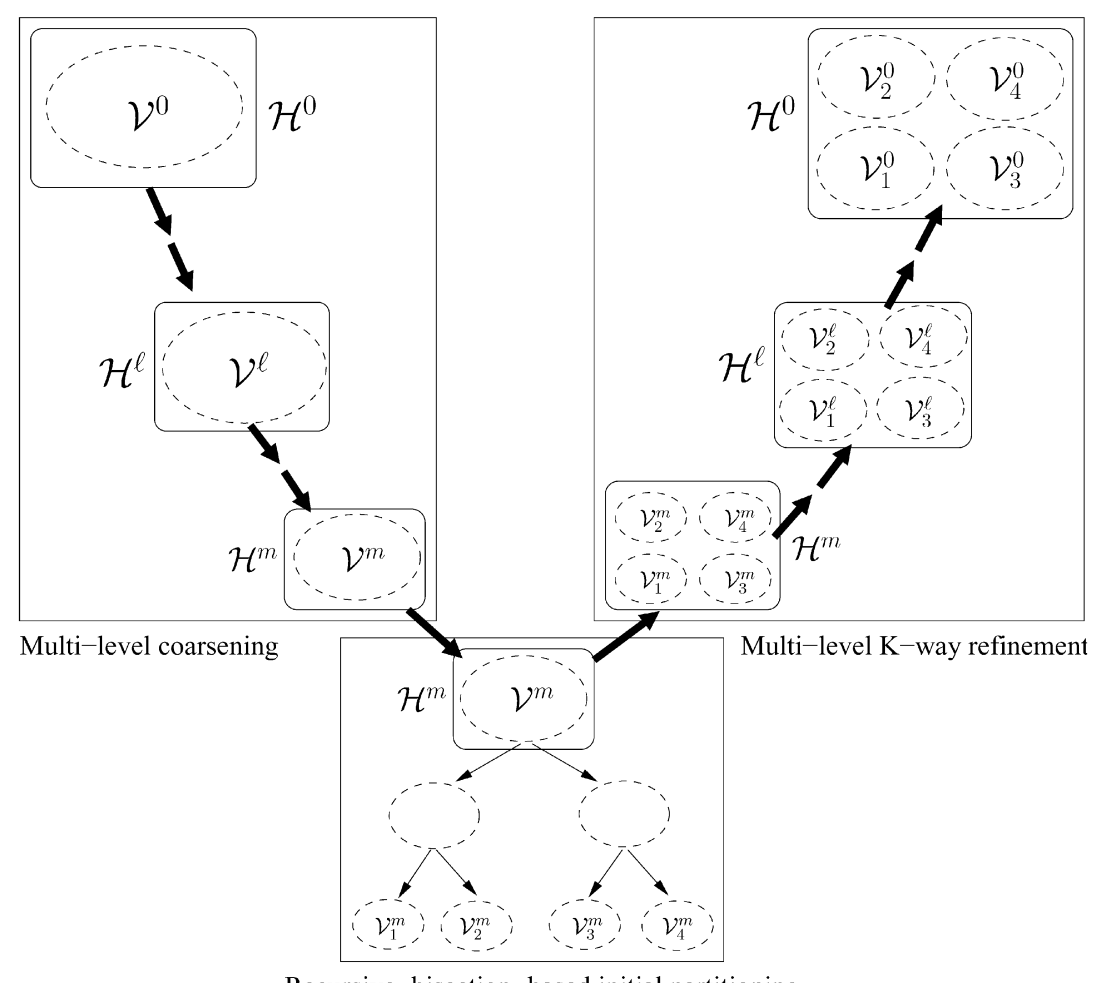

Recursive-bisection-based initial partitioning

Fig. 1. The proposed multi-level $K$-way hypergraph partitioning algorithm. 
nets with $v_{i}$. In the case of nets with variable costs, $v_{i}$ is matched with a vertex $v_{j}$ such that $\sum_{n_{h} \in \mathcal{C}_{i j}} c\left(n_{h}\right)$ is the maximum over all unmatched vertices that share at least one net with $v_{i}$, where $\mathcal{C}_{i j}=\left\{n_{h}: n_{h} \in \operatorname{Nets}\left(v_{i}\right) \wedge n_{h} \in \operatorname{Nets}\left(v_{j}\right)\right\}$, i.e., $\mathcal{C}_{i j}$ denotes the set of nets that connect both $v_{i}$ and $v_{j}$. Each matched vertex pair $\left(v_{i} \in \mathcal{V}^{\ell}, v_{j} \in \mathcal{V}^{\ell}\right)$ forms a single supervertex in $\mathcal{V}^{\ell+1}$.

As the coarsening progresses, nets with identical pin sets may emerge. Such nets become redundant for the subsequent coarser hypergraphs and hence can be removed. In this work, we use an efficient algorithm for identical net detection and elimination. This algorithm is similar to the algorithm in [3], which is later used in [31] for supervariable identification in nested-dissection-based matrix reordering.

\subsection{RB-based initial partitioning}

The objective of the initial partitioning phase is to obtain a $K$-way initial partition $\Pi^{m}=\left\{\mathcal{V}_{1}^{m}, \mathcal{V}_{2}^{m}, \ldots, \mathcal{V}_{K}^{m}\right\}$ of the coarsest hypergraph $\mathcal{H}^{m}$ before direct $K$-way refinement. For this purpose, we use the multi-level RB scheme of $\mathrm{PaToH}$ to partition $\mathcal{H}^{m}$ into $K$ parts. We have observed that it is better to avoid further coarsening within $\mathrm{PaToH}$ since $\mathcal{H}^{m}$ is already coarse enough. At each bisection step of $\mathrm{PaToH}$, we use the greedy hypergraph growing heuristic to bisect the intermediate hypergraphs and the tight boundary FM heuristic [16,17] for refinement. At the end of the initial partitioning phase, if the current imbalance is over the allowed imbalance ratio $\varepsilon$, a balancer, which performs vertex moves (starting with the moves having highest FM gains, i.e., the highest reduction in the cutsize) among the $K$ parts at the expense of an increase in the cutsize, is executed to drop the imbalance below $\varepsilon$.

Although possibilities other than RB exist for generating the initial set of vertex parts, $\mathrm{RB}$ emerges as a viable and practical method. A partition of the coarsest hypergraph $\mathcal{H}^{m}$ generated by RB is very amenable to FM-based refinement since $\mathcal{H}^{m}$ contains nets of small sizes and vertices of high degrees.

\subsection{Multi-level uncoarsening with direct $K$-way refinement}

Every uncoarsening level $\ell$ includes a refinement step, followed by a projection step. In the refinement step, which involves a number of passes, partition $\Pi^{\ell}$ is refined by moving vertices among the $K$ vertex parts, trying to minimize the cutsize while maintaining the balance constraint. In the projection step, the current coarse hypergraph $\mathcal{H}^{\ell}$ and partition $\Pi^{\ell}$ are projected back to $\mathcal{H}^{\ell-1}$ and $\Pi^{\ell-1}$. The refinement and projection steps are iteratively repeated until the top-level, flat hypergraph $\mathcal{H}^{0}$ with a partition $\Pi^{0}$ is obtained. This algorithm is similar to the one described in [36].

At the very beginning of the uncoarsening phase, a connectivity data structure $\Lambda$ and a lookup data structure $\delta$ are created. These structures keep the connectivity of the cut nets to the vertex parts. $\Lambda$ is a $2 \mathrm{D}$ ragged array, where each $1 \mathrm{D}$ array keeps the connectivity set of a cut net. That is, $\Lambda\left(n_{i}\right)$ returns the connectivity set $\Lambda_{i}$ of a cut net $n_{i}$. No information is stored in $\Lambda$ for internal nets. $\delta$ is an $\left|\mathcal{N}_{\text {cut }}\right|$ by $K, 2 \mathrm{D}$ array to lookup the connectivity of a cut net to a part in constant time. That is, $\delta\left(n_{i}, \mathcal{V}_{k}\right)$ returns the number of the pins that cut net $n_{i}$ has in part $\mathcal{V}_{k}$, i.e., $\delta\left(n_{i}, \mathcal{V}_{k}\right)=\left|\operatorname{Pins}\left(n_{i}\right) \cap \mathcal{V}_{k}\right|$.

Both $\Lambda$ and $\delta$ structures are allocated once at the beginning of the uncoarsening phase and maintained during the projection steps. For this purpose, after each coarsening level, a mapping between the nets of the fine and coarse hypergraphs is computed so that $\Lambda$ and $\delta$ arrays are modified appropriately in the corresponding projection steps. Since the pin counts of the nets on the parts may change due to the uncoarsening, the pin counts in the $\delta$ array are updated by iterating over the cut nets in the coarse hypergraph and using the net map created during the corresponding coarsening phase. Similar to the net map, vertex maps are computed in the coarsening steps to be able to determine the part assignments of the vertices in the fine hypergraphs during the projection. Part assignments of vertices are kept in a 1D Part array, where $\operatorname{Part}\left(v_{i}\right)$ shows the current part of vertex $v_{i}$.

During the refinement passes, only boundary vertices are considered for movement. For this purpose, a FIFO queue $B$ of boundary vertices is maintained. A vertex $v_{i}$ is boundary if it is among the pins of at least one cut net $n_{j} . B$ is updated after each vertex move if the move causes some non-boundary vertices to become boundary or some boundary vertices become internal to a part. Each vertex $v_{i}$ has a lock count $b_{i}$, indicating the number of times $v_{i}$ is inserted into $B$. The lock counts are initially set to 0 at the beginning of each refinement pass. Every time a vertex enters $B$, its lock count is incremented by 1 . No vertex $v_{i}$ with a $b_{i}$ value greater than a prespecified threshold is allowed to re-enter $B$. This way, we avoid moving the same vertices repeatedly. The boundary vertex queue $B$ is randomly shuffled at the beginning of each refinement pass.

For vertex movement, each boundary vertex $v_{i} \in B$ is considered in turn. The move of vertex $v_{i}$ is considered only to those parts that are in the union of the connectivity sets of the nets connecting $v_{i}$, excluding the part containing $v_{i}$, if the move satisfies the balance criterion. Note that once the imbalance on part weights is below $\varepsilon$, it is never allowed to rise above this ratio during the direct $K$-way refinement. After gains are computed, the vertex is moved to the part with the highest positive FM gain. Moves with negative FM gains as well as moves with non-positive leave gains are not performed. A refinement pass terminates when queue $B$ becomes empty. No more refinement passes are made if a predetermined pass count is reached or improvement in the cutsize drops below a prespecified threshold.

For FM-based move gain computation for a vertex $v_{i}$, we use the highly efficient algorithm given in Fig. 2. This algorithm first iterates over all nets connecting vertex $v_{i}$ and computes the leave gain for $v_{i}$. If the leave gain is not positive, no further positive move gain is possible and hence the algorithm simply returns. Otherwise, the maximum arrival loss is computed by iterating over all nets connecting $v_{i}$ as well as a separate move gain for all parts (excluding $v_{i}$ 's current part) that are connected by at least one cut net of $v_{i}$. Finally, a total move gain is computed for each part by adding the move gain for the part to the leave gain and subtracting the maximum arrival loss. The 


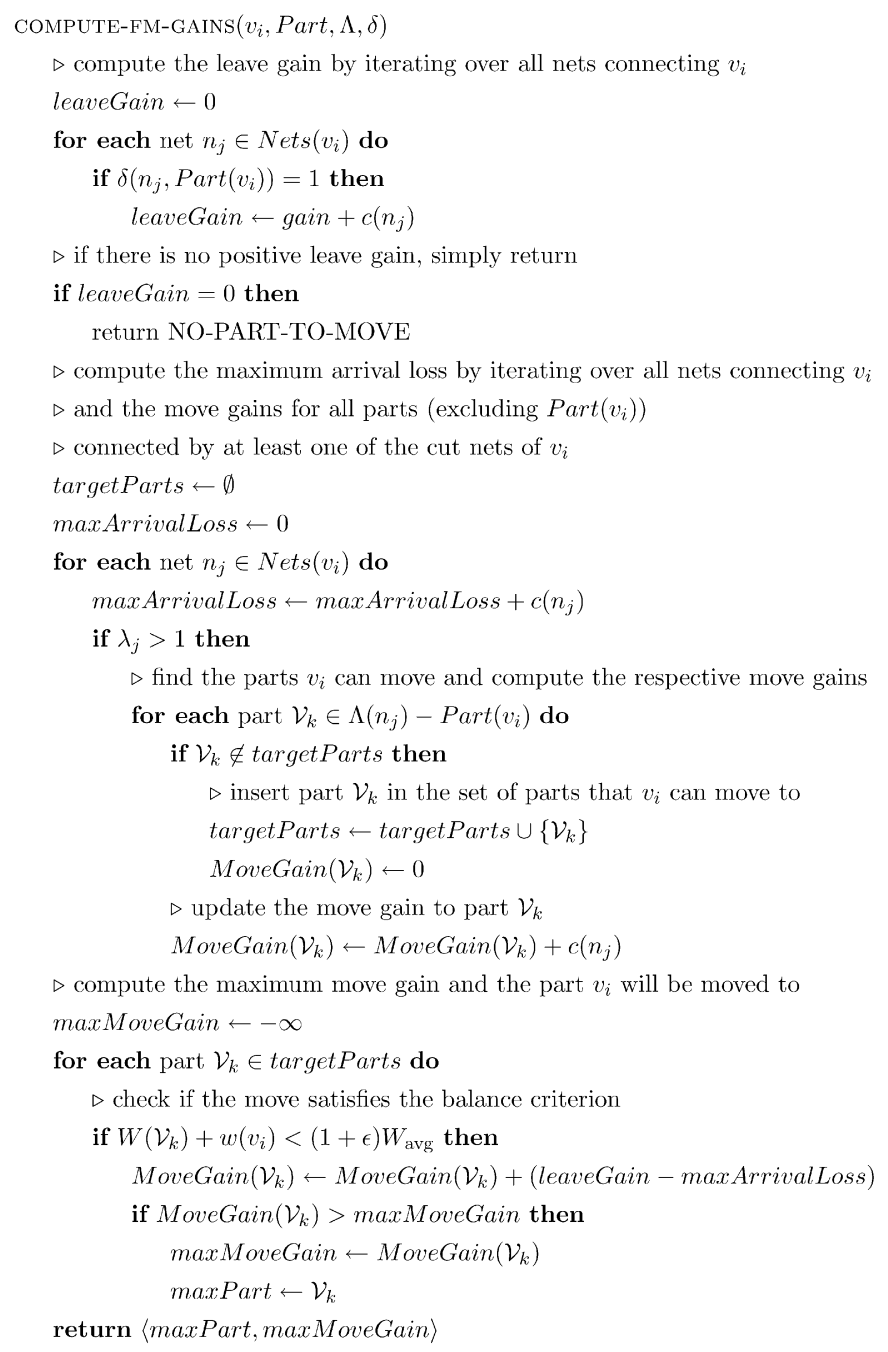

Fig. 2. The algorithm for computing the $K$-way FM gains of a vertex $v_{i}$.

maximum move gain is determined by taking the maximum of the total move gains.

\subsection{Extension to multiple constraints}

Extension to multi-constraint formulation requires verifying the balance constraint for each weight component. As before, zero-gain moves are not performed. During the coarsening phase, the maximum allowed vertex weight is set according to the constraint which has the maximum total vertex weight over all vertices. In the initial partitioning phase, the multi-constraint RB-based partitioning feature of $\mathrm{PaToH}$ is used with default parameters to obtain an initial $K$-way partition.

\section{Extensions to hypergraphs with fixed vertices}

Our extension to partitioning hypergraphs with fixed vertices follows the multi-level paradigm, which is, in our case, composed of three phases: coarsening with modified heavyconnectivity matching, initial partitioning with maximumweighted bipartite graph matching, and $K$-way direct refinement with locked fixed vertices. Throughout the presentation, we assume that, at each coarsening/uncoarsening level $\ell$, $f_{i}^{\ell}$ is a fixed vertex in the set $\mathcal{F}^{\ell}$ of fixed vertices, and $o_{j}^{\ell}$ is an ordinary vertex in the set $\mathcal{O}^{\ell}$ of ordinary vertices, where $\mathcal{O}^{\ell}=\mathcal{V}^{\ell}-\mathcal{F}^{\ell}$. For each part $\mathcal{V}_{k}^{0}$, there is a set $\mathcal{F}_{k}^{0}$ of fixed vertices that must end up in $\mathcal{V}_{k}^{0}$ at the end of the partitioning such that $\mathcal{F}^{0}=\mathcal{F}_{1}^{0} \cup \mathcal{F}_{2}^{0} \cdots \cup \mathcal{F}_{K}^{0}$. We also assume that the weights of the fixed vertex sets are fairly balanced.

For the coarsening phase of our algorithm, we modify the heavy-connectivity matching heuristic such that no two fixed vertices $f_{i}^{\ell} \in \mathcal{F}^{\ell}$ and $f_{j}^{\ell} \in \mathcal{F}^{\ell}$ are matched at any coarsening level $\ell$. However, any fixed vertex $f_{i}^{\ell}$ in a fixed vertex set $\mathcal{F}_{k}^{\ell}$ can be matched with an ordinary vertex $o_{j}^{\ell} \in \mathcal{O}^{\ell}$, forming a fixed supervertex $f_{i}^{\ell+1}$ in $\mathcal{F}_{k}^{\ell+1}$. Ordinary vertices are matched as before. Consequently, fixed vertices are propagated throughout the coarsening such that $\left|\mathcal{F}_{k}^{\ell+1}\right|=\left|\mathcal{F}_{k}^{\ell}\right|$, for $k=1,2, \ldots, K$ and $\ell=0,1, \ldots, m-1$. Hence, in the coarsest hypergraph $\mathcal{H}^{m}$, there are $\left|\mathcal{F}^{m}\right|=\left|\mathcal{F}^{0}\right|$ fixed supervertices.

In the initial partitioning phase, a hypergraph $\tilde{\mathcal{H}}^{m}=\left(\mathcal{O}^{m}\right.$, $\tilde{\mathcal{N}}^{m}$ ) that is free from fixed vertices is formed by temporarily removing fixed supervertices from $\mathcal{H}^{m}$. In $\tilde{\mathcal{H}}^{m}, \tilde{\mathcal{N}}^{m}$ is a subset of nets in $\mathcal{N}^{m}$ whose pins contain at least two ordinary vertices, i.e., $\tilde{\mathcal{N}}^{m}=\left\{n_{i}^{m}: n_{i}^{m} \in \mathcal{N}^{m} \wedge\left|\mathcal{O}^{m} \cap \operatorname{Pins}\left(n_{i}^{m}\right)\right|>1\right\}$. Note that the nets that connect only one ordinary vertex are not retained in $\tilde{\mathcal{H}}^{m}$ since single-pin nets do not contribute to the cutsize at all. After $\tilde{\mathcal{H}}^{m}$ is formed, it is partitioned to obtain a $K$-way vertex partition $\tilde{\Pi}^{m}=\left\{\mathcal{O}_{1}^{m}, \mathcal{O}_{2}^{m}, \ldots, \mathcal{O}_{K}^{m}\right\}$ over the set $\mathcal{O}^{m}$ of ordinary vertices. Partition $\tilde{\Pi}^{m}$ induces an initial part assignment for each ordinary vertex in $\mathcal{V}^{m}$, i.e., $o_{i}^{m} \in \mathcal{O}_{k}^{m} \Rightarrow$ $\operatorname{Part}\left(v_{i}^{m}\right)=\mathcal{V}_{k}^{m}$. However, this initial assignment induced by $\tilde{\Pi}^{m}$ may not be appropriate in terms of the cutsize since fixed vertices are not considered at all in computation of the cutsize. Note that cutsize $\left(\tilde{\Pi}^{m}\right)$ is a lower bound on cutsize $\left(\Pi^{m}\right)$. A net $n_{j}$ has the potential to increase the cutsize by its cost times the number of fixed vertex parts that it connects, i.e., by $c\left(n_{j}^{m}\right) \bar{\lambda}_{j}^{m}$, where $\bar{\lambda}_{j}^{m}=\left|\bar{\Lambda}_{j}^{m}\right|=\left|\left\{\mathcal{F}_{k}^{m}: \operatorname{Pins}\left(n_{j}^{m}\right) \cap \mathcal{F}_{k}^{m} \neq \emptyset\right\}\right|$. Therefore, $U=\operatorname{cutsize}\left(\tilde{\Pi}^{m}\right)+\sum_{n_{j}^{m}} c\left(n_{j}^{m}\right) \bar{\lambda}_{j}^{m}$ is an upper bound on cutsize $\left(\Pi^{m}\right)$. At this point, a relabeling of ordinary vertex parts must be found so that the cutsize is tried to be minimized as the fixed vertices are assigned to appropriate parts. We formulate this relabeling problem as a maximum-weighted bipartite graph matching problem [12]. This formulation is valid for any number of fixed vertices.

In the proposed formulation, the sets of fixed supervertices and the ordinary vertex parts form the two node sets of a bipartite graph $\mathcal{B}=(\mathcal{X}, \mathcal{Y})$. That is, in $\mathcal{B}$, for each fixed vertex set $\mathcal{F}_{k}^{m}$, there exists a node $x_{k} \in \mathcal{X}$, and for each ordinary vertex part $\mathcal{O}_{\ell}^{m}$ of $\tilde{\Pi}^{m}$, there exists a node $y_{\ell} \in \mathcal{Y}$. The bipartite graph contains all possible $\left(x_{k}, y_{\ell}\right)$ edges, initially with zero weights. The weight of an edge $\left(x_{k}, y_{\ell}\right)$ is increased by the cost of every net that connects at least one vertex in both $\mathcal{F}_{k}^{m}$ and $\mathcal{O}_{\ell}^{m}$. That is, a net $n_{j}^{m}$ increases the weight of edge $\left(x_{k}, y_{\ell}\right)$ by its cost $c\left(n_{j}^{m}\right)$ if and only if $\operatorname{Pins}\left(n_{j}^{m}\right) \cap \mathcal{F}_{k}^{m} \neq \emptyset$ and $\operatorname{Pins}\left(n_{j}^{m}\right) \cap \mathcal{O}_{\ell}^{m} \neq \emptyset$. This weight on edge $\left(x_{k}, y_{\ell}\right)$ corresponds to a saving of $c\left(n_{j}^{m}\right)$ from upper bound $U$ if $\mathcal{F}_{k}^{m}$ is matched with $\mathcal{O}_{\ell}^{m}$. 
a

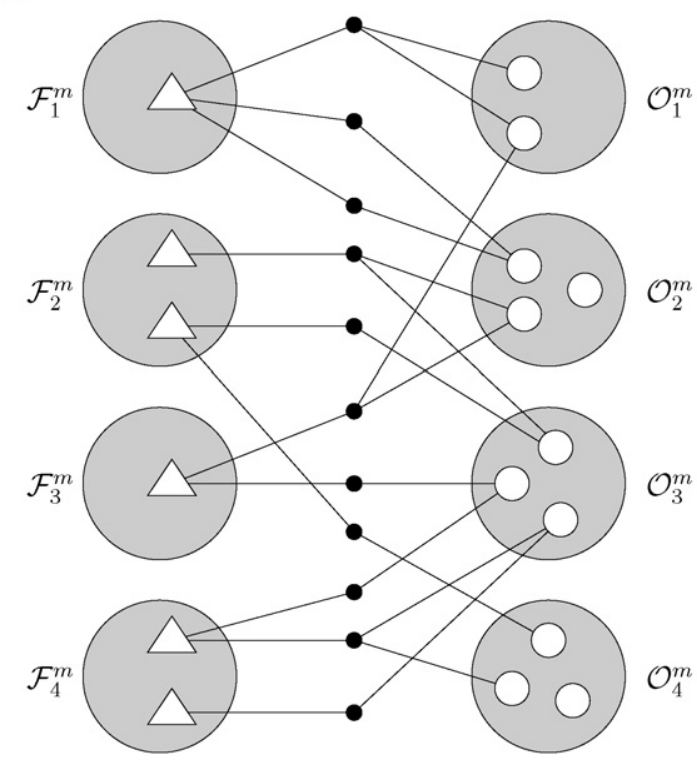

b

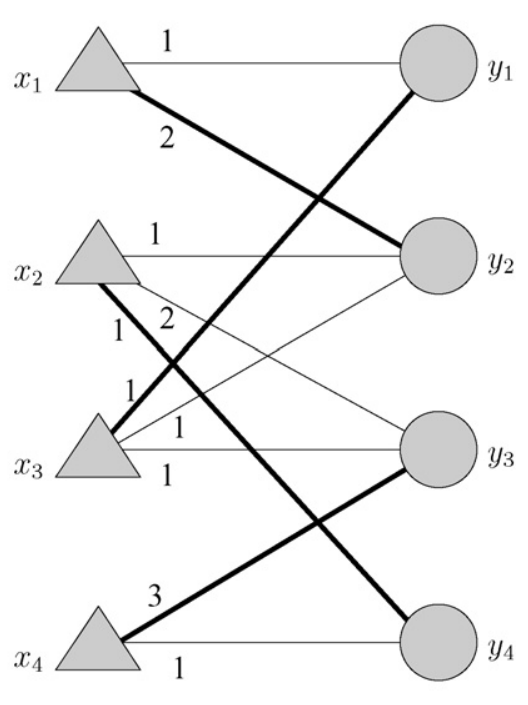

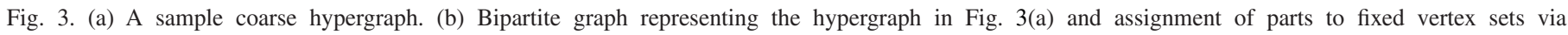
maximum-weighted matching shown by bold edges.

In this setting, finding the maximum-weighted matching in bipartite graph $\mathcal{B}$ corresponds to finding a matching between fixed vertex sets and ordinary vertex parts which has the minimum increase in the cutsize when fixed vertices are re-introduced into $\tilde{\mathcal{H}}^{m}$. Each edge $\left(x_{k}, y_{\ell}\right)$ in the resulting maximum-weighted matching $\mathcal{M}$ matches a fixed vertex set to an ordinary vertex part. Using $\mathcal{M}$, ordinary vertex parts are relabeled. Vertices in $\mathcal{O}_{\ell}^{m}$ are reassigned to part $\mathcal{V}_{k}^{m}$ if and only if edge $\left(x_{k}, y_{\ell}\right)$ is in $\mathcal{M}$. Note that the fixed vertices in $\mathcal{F}_{k}^{m}$ are in part $\mathcal{V}_{k}^{m}$ and hence the partition conforms to the given partition on fixed vertices. This relabeling induces an initial partition $\Pi^{m}$. Here, $\operatorname{cutsize}\left(\Pi^{m}\right)=U-\operatorname{weight}(\mathcal{M})$, where $\operatorname{weight}(\mathcal{M})$ is the weight of matching $\mathcal{M}$ and is equal to the saving on the cutsize. Since $\mathcal{M}$ is the maximum-weighted matching, this defines an optimum solution for the relabeling problem.

Fig. 3(a) shows a sample, coarse hypergraph $\mathcal{H}^{m}$, where fixed and ordinary vertices are, respectively, represented as triangles and circles. For ease of presentation, unit net costs are assumed and only the nets connecting the fixed vertices and ordinary vertices are displayed since all cost contribution on the edges of the constructed bipartite graph are due to such nets. Note that, in this example, the upper bound on cutsize $\left(\Pi^{m}\right)$ is $U=3+$ $11=14$. The linear assignment of fixed vertex sets to ordinary vertex parts (i.e., $\mathcal{F}_{k}^{m}$ matched with $\mathcal{O}_{k}^{m}$, for $k=1,2, \ldots, K$ ) has a cost saving of $\operatorname{weight}(\mathcal{M})=1+1+1+1=4$. Hence, cutsize $\left(\Pi^{m}\right)=U-\operatorname{weight}(\mathcal{M})=14-4=10$.

Fig. 3(b) displays the bipartite graph constructed for the sample hypergraph in Fig. 3(a), without displaying the zero-weight edges for clarity. In this figure, triangles and circles denote the sets of fixed vertices and ordinary vertex parts, respectively. As seen in Fig. 3(b), there exists an edge $\left(x_{2}, y_{3}\right)$ with a weight of 2. This is because two unit-cost nets connect $\mathcal{F}_{2}^{m}$ and $\mathcal{O}_{3}^{m}$. In the figure, the set of bold edges shows the maximum-weighted matching $\mathcal{M}=\left\{\left(x_{1}, y_{2}\right),\left(x_{2}, y_{4}\right),\left(x_{3}, y_{1}\right),\left(x_{4}, y_{3}\right)\right\}$, which assigns the vertices in $\mathcal{F}_{1}^{m}, \mathcal{F}_{2}^{m}, \mathcal{F}_{3}^{m}$, and $\mathcal{F}_{4}^{m}$ to $\mathcal{O}_{2}^{m}, \mathcal{O}_{4}^{m}, \mathcal{O}_{1}^{m}$, and $\mathcal{O}_{3}^{m}$, respectively. As seen in the figure, matching $\mathcal{M}$ obtains the highest possible cost saving of weight $(\mathcal{M})=2+1+1+3=7$. Hence, $\operatorname{cutsize}\left(\Pi^{m}\right)=U-\operatorname{weight}(\mathcal{M})=14-7=7$. This cutsize is $10-7=3$ less than the cutsize achieved by linear assignment.

During the $K$-way refinement phase, $\Pi^{m}$ is refined using a modified version of the algorithm described in Section 3.3. Throughout the uncoarsening, the fixed vertices are locked to their parts and are not allowed to move between the parts. Hence, each fixed vertex $f_{i}^{0}$ whose corresponding supervertex in the $m$ th level is $f_{i}^{m}$ ends up in part $\mathcal{V}_{k}^{0}$ if and only if $f_{i}^{m} \in \mathcal{F}_{k}$.

\section{Experiments}

\subsection{Experimental platform}

In the experiments, a Pentium IV $3.00 \mathrm{GHz}$ PC with $1 \mathrm{~GB}$ of main memory, $512 \mathrm{~KB}$ of $\mathrm{L} 2$ cache, and $8 \mathrm{~KB}$ of $\mathrm{L} 1$ cache is used. All algorithms are implemented in $\mathrm{C}$ and are compiled in gcc with -O3 optimization option. Due to the randomized nature of some of the heuristics, the results are reported by averaging the values obtained in 20 different runs, each randomly seeded.

The hypergraphs used in the experiments are the row-net hypergraphs [17] of some widely used square sparse matrices obtained from the University of Florida Sparse Matrix Collection [21]. The properties of the hypergraphs are given in Table 1, where the hypergraphs are sorted in increasing order of the number of pins. In all hypergraphs, the number of nets is equal to the number of vertices, and the average vertex degree is equal to the average net size since all matrices are square matrices. Since the internal data structures maintained during 
Table 1

Properties of the hypergraphs used in the experiments

\begin{tabular}{lrrr}
\hline Data set & \# of vertices & \# of pins & Avg. net size \\
\hline dawson5 & 51,537 & $1,010,777$ & 19.61 \\
language & 399,130 & $1,216,334$ & 3.05 \\
Lin & 256,000 & $1,766,400$ & 6.90 \\
poisson3Db & 85,623 & $2,374,949$ & 27.74 \\
helm2d03 & 392,257 & $2,741,935$ & 6.99 \\
stomach & 213,360 & $3,021,648$ & 14.16 \\
barrier2-1 & 113,076 & $3,805,068$ & 33.65 \\
Hamr1e3 & $1,447,360$ & $5,514,242$ & 3.81 \\
pre2 & 659,033 & $5,959,282$ & 9.04 \\
cage13 & 445,135 & $7,479,343$ & 16.80 \\
hood & 220,542 & $10,768,436$ & 48.83 \\
bmw3_2 & 227,362 & $11,288,630$ & 49.65 \\
\hline
\end{tabular}

the partitioning do not fit into the memory for the Hamrle3, cage13, and pre2 data sets, they are partitioned on a PC with 2 GB main memory, all other parameters remaining the same. We compare the proposed algorithm, referred to here as $\mathrm{kPaToH}$, with $\mathrm{PaToH}$ [16] for two reasons. First, the implementation of $\mathrm{kPaToH}$ is based on $\mathrm{PaToH}$. Second, in previous experiments (e.g., see [14,27]), PaToH was found to be performing better than the other hypergraph partitioners.

In the tables, the minimum cutsizes (Min cutsize) and average cutsizes (Avg cutsize) achieved by both partitioners are reported over all data sets together with their average partitioning times (Avg time), for varying number $K$ of parts, where $K \in\{32,64,128,256,512\}$. The rightmost two columns in Tables 2, 5, 6, and 8 show the percent average cutsize improvement (\%Cutsize) and the speedup (Spdup) of kPaToH over PaToH. The averages over all data sets are displayed as a separate entry at the bottom of these tables. Unless otherwise stated, the maximum number of $K$-way refinement passes in $\mathrm{kPaToH}$ is set to 3. Since identical net elimination brings around $5 \%$ improvement on the execution time of $\mathrm{kPaToH}$ but no speedup on $\mathrm{PaToH}$, we run both $\mathrm{PaToH}$ and $\mathrm{kPaToH}$ without identical net elimination for a fair comparison. In single-constraint partitioning, weight $w^{1}\left(v_{i}\right)$ of a vertex $v_{i}$ is set equal to its vertex degree $d\left(v_{i}\right)$, i.e., $w^{1}\left(v_{i}\right)=d\left(v_{i}\right)$. The allowed imbalance threshold is set to $10 \%$ and is met in all experiments. PaToH is executed with default options.

\subsection{Experiments on standard hypergraph partitioning}

Table 2 displays the performance comparison of $\mathrm{PaToH}$ and $\mathrm{kPaToH}$ for standard hypergraph partitioning. According to the averages over all data sets, as $K$ increases, $\mathrm{kPaToH}$ begins to perform better in reducing the cutsize compared to $\mathrm{PaToH}$. The average cutsize improvement of $4.82 \%$ at $K=32$ rises to $6.81 \%$ at $K=256$. A similar behavior is observed in the improvement of $\mathrm{kPaToH}$ over $\mathrm{PaToH}$ in the minimum cutsizes achieved. In the speedups $\mathrm{kPaToH}$ obtains over $\mathrm{PaToH}$, a slight decrease is observed as $K$ increases. However, even at $K=256, \mathrm{kPaToH}$ runs 1.62 times faster than $\mathrm{PaToH}$ and is 1.78 times faster on the overall average.
According to Table 2, except for a single case (the language data set with $K=32$ ), $\mathrm{kPaToH}$ achieves lower cutsizes than $\mathrm{PaToH}$ for all data sets and $K$ values. In general, $\mathrm{kPaToH}$ performs relatively better in reducing the cutsize on hypergraphs having average net sizes between 6 and 20. This is expected since $\mathrm{PaToH}$ is already very effective in partitioning hypergraphs with low net sizes (e.g., language and Hamrle3). On the other hand, in partitioning hypergraphs with very large net sizes (e.g., barrier2-1 and bmw3_2), the performance gap between the partitioners begins to decrease. This is mainly due to performing only the moves with positive gains. Such moves are rare when the nets are highly connected to the parts.

Tables 3 and 4 show the overall percent execution time dissection of the $\mathrm{PaToH}$ and $\mathrm{kPaToH}$ algorithms, respectively. The tables further display the percent execution time dissection of coarsening and uncoarsening phases for both algorithms. These experiments are conducted on three data sets (language, pre2, and hood) each with different average net size characteristics $(3.05,9.04$, and 48.83 , respectively), for $K=32$ and 256. The dissections of both $\mathrm{PaToH}$ and $\mathrm{kPaToH}$ algorithms are given according to the traditional multi-level partitioning paradigm, which involves an initialization phase followed by the coarsening, initial partitioning, and uncoarsening phases. In case of $\mathrm{PaToH}$, these phases are repeated for each hypergraph produced via bisection, and hence the cost of splitting the hypergraph into two after bisections is also considered.

According to Tables 3 and 4, the main overhead of $\mathrm{PaToH}$ is at the coarsening step, whereas the percent overhead of uncoarsening is relatively more dominant in case of $\mathrm{kPaToH}$. In general, as $K$ increases from 32 to 256, percent uncoarsening and splitting overheads of PaToH slightly increase. In case of $\mathrm{kPaToH}$, the $K$-way initial partitioning phase is what most suffers from large $K$ values. In $\mathrm{kPaToH}$, the behavior of the uncoarsening phase is data set dependent. As the average net size increases, the percent overhead of uncoarsening begins to increase with increasing $K$. This is because the refinement step, which takes the most of the uncoarsening time, is not affected by changing $K$ if the average net size is low as in the case of the language data set. In the hood data set, the increase in the percent overhead of the uncoarsening phase from $27.1 \%$ to $57.7 \%$ as $K$ goes from 32 to 256 is due to the increase in the solution space, which prevents quick termination of the refinement phase.

\subsection{Experiments on multi-constraint partitioning}

Tables 5 and 6 show the performance of $\mathrm{PaToH}$ and $\mathrm{kPaToH}$ in multi-constraint partitioning ( 2 and 4 constraints, respectively). In the 2-constraint case, a unit weight is used as the second vertex weight for all vertices, i.e., $w^{2}\left(v_{i}\right)=1$, in addition to the first vertex weight $w^{1}\left(v_{i}\right)=d\left(v_{i}\right)$. In the 4-constraint case, random integer weights $w^{3}\left(v_{i}\right)=\alpha_{i}$, where $1 \leqslant \alpha_{i} \leqslant w^{1}\left(v_{i}\right)-1$, and $w^{4}\left(v_{i}\right)=w^{1}\left(v_{i}\right)-\alpha_{i}$ are, respectively, used as the third and fourth vertex weights.

As seen from Tables 5 and $6, \mathrm{kPaToH}$ performs much better than PaToH. A comparison of Tables 2, 5, and 6 shows 
Table 2

Performance of $\mathrm{PaToH}$ and $\mathrm{kPaToH}$ in partitioning hypergraphs with a single partitioning constraint and no fixed vertices

\begin{tabular}{|c|c|c|c|c|c|c|c|c|c|}
\hline \multirow[t]{2}{*}{ Data set } & \multirow[t]{2}{*}{$K$} & \multicolumn{2}{|l|}{ Min. cutsize } & \multicolumn{2}{|l|}{ Avg. cutsize } & \multicolumn{2}{|c|}{ Avg. time } & \multicolumn{2}{|c|}{ Improvement } \\
\hline & & $\mathrm{PaToH}$ & $\mathrm{kPaToH}$ & $\mathrm{PaToH}$ & $\mathrm{kPaToH}$ & $\mathrm{PaToH}$ & $\mathrm{kPaToH}$ & $\%$ Cutsize & Spdup \\
\hline \multirow{3}{*}{ dawson5 } & 32 & 6,959 & 6,286 & 7,468 & 6,907 & 1.524 & 0.715 & 7.51 & 2.13 \\
\hline & 128 & 19,058 & 17,140 & 19,393 & 17,767 & 2.099 & 1.291 & 8.39 & 1.63 \\
\hline & 256 & 29,655 & 28,035 & 30,351 & 28,396 & 2.380 & 1.762 & 6.44 & 1.35 \\
\hline & 128 & 119,636 & 117,781 & 120,234 & 119,184 & 13.835 & 9.992 & 0.87 & 1.38 \\
\hline & 256 & 131,251 & 130,679 & 131,690 & 131,526 & 14.489 & 10.303 & 0.12 & 1.41 \\
\hline \multirow[t]{4}{*}{ Lin } & 32 & 49,458 & 43,926 & 50,800 & 44,733 & 5.763 & 4.751 & 11.94 & 1.21 \\
\hline & 64 & 68,994 & 60,107 & 70,645 & 60,832 & 6.632 & 5.505 & 13.89 & 1.20 \\
\hline & 128 & 91,701 & 79,910 & 93,622 & 80,878 & 7.471 & 6.510 & 13.61 & 1.15 \\
\hline & 256 & 119,529 & 105,567 & 121,346 & 105,916 & 8.327 & 7.942 & 12.72 & 1.05 \\
\hline & 256 & 121,733 & 114,384 & 123,051 & 116,147 & 12.240 & 11.738 & 5.61 & 1.04 \\
\hline \multirow[t]{4}{*}{ helm2d03 } & 32 & 13,016 & 12,487 & 13,591 & 12,965 & 7.689 & 2.845 & 4.61 & 2.70 \\
\hline & 64 & 19,677 & 18,841 & 20,251 & 19,236 & 8.757 & 3.228 & 5.01 & 2.71 \\
\hline & 128 & 29,169 & 27,660 & 29,696 & 28,096 & 9.801 & 3.790 & 5.38 & 2.59 \\
\hline & 256 & 42,763 & 40,517 & 43,079 & 40,950 & 10.850 & 4.717 & 4.94 & 2.30 \\
\hline \multirow[t]{4}{*}{ stomach } & 32 & 26,231 & 25,757 & 27,054 & 26,184 & 6.635 & 3.327 & 3.22 & 1.99 \\
\hline & 64 & 37,885 & 36,732 & 38,918 & 37,113 & 7.795 & 4.097 & 4.64 & 1.90 \\
\hline & 128 & 54,651 & 52,150 & 55,370 & 52,817 & 8.968 & 5.175 & 4.61 & 1.73 \\
\hline & 256 & 78,289 & 74,863 & 79,143 & 75,572 & 10.156 & 6.774 & 4.51 & 1.50 \\
\hline \multirow[t]{2}{*}{ barrier2-1 } & 32 & 52,877 & 51,472 & 53,560 & 52,623 & 9.797 & 7.292 & 1.75 & 1.34 \\
\hline & 64 & 73,864 & 71,879 & 75,037 & 73,149 & 11.135 & 8.609 & 2.52 & 1.29 \\
\hline \multirow[t]{4}{*}{ pre2 } & 32 & 82,591 & 75,860 & 85,456 & 80,238 & 24.406 & 15.070 & 6.11 & 1.62 \\
\hline & 64 & 108,714 & 99,609 & 112,486 & 105,476 & 28.484 & 16.929 & 6.23 & 1.68 \\
\hline & 128 & 139,605 & 120,469 & 143,879 & 122,822 & 32.250 & 18.071 & 14.64 & 1.78 \\
\hline & 256 & 177,310 & 137,899 & 183,037 & 141,091 & 35.702 & 19.743 & 22.92 & 1.81 \\
\hline \multirow[t]{4}{*}{ cage13 } & 32 & 369,330 & 339,563 & 373,617 & 345,740 & 45.887 & 45.590 & 7.46 & 1.01 \\
\hline & 64 & 490,789 & 448,407 & 497,744 & 455,056 & 51.035 & 49.528 & 8.58 & 1.03 \\
\hline & 128 & 643,278 & 584,178 & 647,609 & 589,316 & 55.754 & 52.972 & 9.00 & 1.05 \\
\hline & 256 & 824,294 & 749,315 & 829,962 & 752,394 & 59.928 & 56.450 & 9.35 & 1.06 \\
\hline \multirow[t]{4}{*}{ hood } & 32 & 22,799 & 22,204 & 24,392 & 23,041 & 15.693 & 5.386 & 5.54 & 2.91 \\
\hline & 64 & 37,877 & 37,058 & 39,855 & 38,239 & 18.383 & 6.607 & 4.05 & 2.78 \\
\hline & 128 & 60,039 & 56,903 & 61,087 & 58,198 & 20.983 & 8.073 & 4.73 & 2.60 \\
\hline & 256 & 91,007 & 86,009 & 92,367 & 87,284 & 23.515 & 10.303 & 5.50 & 2.28 \\
\hline bmw3_2 & 32 & 29,861 & 28,298 & 31,129 & 29,792 & 15.383 & 5.545 & 4.30 & 2.77 \\
\hline & 64 & 44,208 & 42,465 & 45,376 & 43,820 & 18.150 & 6.682 & 3.43 & 2.72 \\
\hline & 128 & 65,752 & 63,652 & 67,551 & 64,956 & 20.853 & 8.065 & 3.84 & 2.59 \\
\hline & 256 & 100,504 & 97,714 & 102,548 & 99,341 & 23.454 & 10.196 & 3.13 & 2.30 \\
\hline AVERAGE & 32 & 1.000 & 0.950 & 1.000 & 0.952 & 1.000 & 0.606 & 4.82 & 1.88 \\
\hline & 64 & 1.000 & 0.947 & 1.000 & 0.945 & 1.000 & 0.611 & 5.47 & 1.85 \\
\hline & 128 & 1.000 & 0.938 & 1.000 & 0.938 & 1.000 & 0.633 & 6.18 & 1.77 \\
\hline & 256 & 1.000 & 0.934 & 1.000 & 0.932 & 1.000 & 0.679 & 6.81 & 1.62 \\
\hline
\end{tabular}


Table 3

Percent dissection of $\mathrm{PaToH}$ execution time

\begin{tabular}{|c|c|c|c|c|c|c|}
\hline \multirow[t]{2}{*}{ Execution phase } & \multicolumn{2}{|c|}{ language } & \multicolumn{2}{|l|}{ pre2 } & \multicolumn{2}{|l|}{ hood } \\
\hline & $K=32$ & $K=256$ & $K=32$ & $K=256$ & $K=32$ & $K=256$ \\
\hline Initialization & 1.0 & 0.9 & 1.1 & 0.8 & 2.1 & 1.4 \\
\hline Coarsening & 73.5 & 70.9 & 84.5 & 82.8 & 86.3 & 84.6 \\
\hline Visit order computation & 0.8 & 1.2 & 0.5 & 0.6 & 0.2 & 0.3 \\
\hline Vertex matching & 72.3 & 70.0 & 73.6 & 74.2 & 80.7 & 81.4 \\
\hline Vertex mapping & 2.2 & 2.7 & 1.1 & 1.1 & 0.5 & 0.5 \\
\hline Hypergraph construction & 24.7 & 26.1 & 24.8 & 24.1 & 18.6 & 17.8 \\
\hline Initial partitioning & 1.9 & 1.9 & 1.0 & 1.3 & 0.4 & 0.9 \\
\hline Uncoarsening & 19.1 & 20.6 & 9.5 & 10.6 & 4.6 & 5.8 \\
\hline Initial gain computations & 27.0 & 27.0 & 32.8 & 30.7 & 19.9 & 20.1 \\
\hline Refinement & 44.2 & 43.4 & 17.4 & 24.1 & 18.5 & 29.4 \\
\hline Projection & 28.8 & 29.6 & 49.8 & 45.2 & 61.6 & 50.5 \\
\hline Splitting & 4.5 & 5.7 & 3.9 & 4.5 & 6.6 & 7.3 \\
\hline
\end{tabular}

Table 4

Percent dissection of $\mathrm{kPaToH}$ execution time

\begin{tabular}{|c|c|c|c|c|c|c|}
\hline \multirow[t]{2}{*}{ Execution phase } & \multicolumn{2}{|c|}{ language } & \multicolumn{2}{|l|}{ pre2 } & \multicolumn{2}{|l|}{ hood } \\
\hline & $K=32$ & $K=256$ & $K=32$ & $K=256$ & $K=32$ & $K=256$ \\
\hline Initialization & 2.9 & 2.8 & 3.7 & 2.8 & 12.0 & 6.4 \\
\hline Coarsening & 45.2 & 38.5 & 32.1 & 22.6 & 56.8 & 28.8 \\
\hline Visit order computation & 0.4 & 0.4 & 0.6 & 0.6 & 0.2 & 0.2 \\
\hline Vertex matching & 81.0 & 84.5 & 70.5 & 73.5 & 76.7 & 77.9 \\
\hline Vertex mapping & 1.3 & 1.3 & 1.2 & 1.2 & 0.5 & 0.6 \\
\hline Hypergraph construction & 17.3 & 13.8 & 27.7 & 24.7 & 22.6 & 21.3 \\
\hline Initial partitioning & 4.5 & 11.3 & 4.4 & 11.8 & 4.1 & 7.1 \\
\hline Uncoarsening & 47.4 & 47.4 & 59.8 & 62.8 & 27.1 & 57.7 \\
\hline Initialization & 1.5 & 6.2 & 1.3 & 5.4 & 1.5 & 4.3 \\
\hline Refinement & 88.3 & 82.7 & 91.6 & 84.8 & 87.9 & 85.6 \\
\hline Projection & 10.2 & 11.1 & 7.1 & 9.8 & 10.6 & 10.1 \\
\hline
\end{tabular}

that increasing number of partitioning constraints favors $\mathrm{kPa}$ $\mathrm{ToH}$. On average, the cutsize improvement of $\mathrm{kPaToH}$ over $\mathrm{Pa}-$ $\mathrm{ToH}$, which is $5.82 \%$ in the single-constraint case, increases to $20.98 \%$ in the 2-constraint case and further increases to $40.02 \%$ in the 4-constraint case. In other words, the degradation in the solution qualities of $\mathrm{kPaToH}$ is considerably less than that of $\mathrm{PaToH}$ as the number of constraints increases.

The speedups, although being slightly lower, are close to the speedups in the single-constraint case. This slight decrease stems from the fact that the overhead that multi-constraint partitioning introduces to the FM-based refinement algorithm is higher in $\mathrm{kPaToH}$ compared to $\mathrm{PaToH}$.

\subsection{Experiments on partitioning with fixed vertices}

In experiments on partitioning hypergraphs with fixed vertices, we use hypergraphs emerging in a real-life problem [10]. The properties of the hypergraphs are given in Table 7. In naming the data sets, the numbers after the dash indicate the number $F$ of fixed vertices in the hypergraph, e.g., there are $F=32$ fixed vertices in the BF-32 data set. In the experiments, each part is assigned an equal number of fixed vertices. In $\mathrm{CC}$ data sets, the net size variation is low, whereas, in BF and OP data sets, the net sizes show high variation.

Table 8 illustrates the performance results obtained in partitioning hypergraphs with fixed vertices. These results are impressive as $\mathrm{kPaToH}$ outperforms $\mathrm{PaToH}$ by up to $31.28 \%$ in reducing the cutsize. On the overall average, $\mathrm{kPaToH}$ incurs a lower cutsize (between $17.09 \%$ and $21.07 \%$ ) than $\mathrm{PaToH}$. At the same time, $\mathrm{kPaToH}$ is around 2.3 times faster on the average.

In general, $\mathrm{kPaToH}$ shows better cutsize performance than PaToH as $K$ decreases and $F$ increases. This is due to the fact that the disability of $\mathrm{PaToH}$ to recursively bisect fixed vertices between two parts in a way that will lead to better cutsizes in the following bisections becomes more pronounced if the number of fixed vertices per part is high. In general, compared to $\mathrm{PaToH}$, the relative performance of $\mathrm{kPaToH}$ in minimizing the cutsize is better in BF and OP data sets, which have high variation in net sizes. 
Table 5

Performance of $\mathrm{PaToH}$ and $\mathrm{kPaToH}$ in partitioning hypergraphs with two partitioning constraints

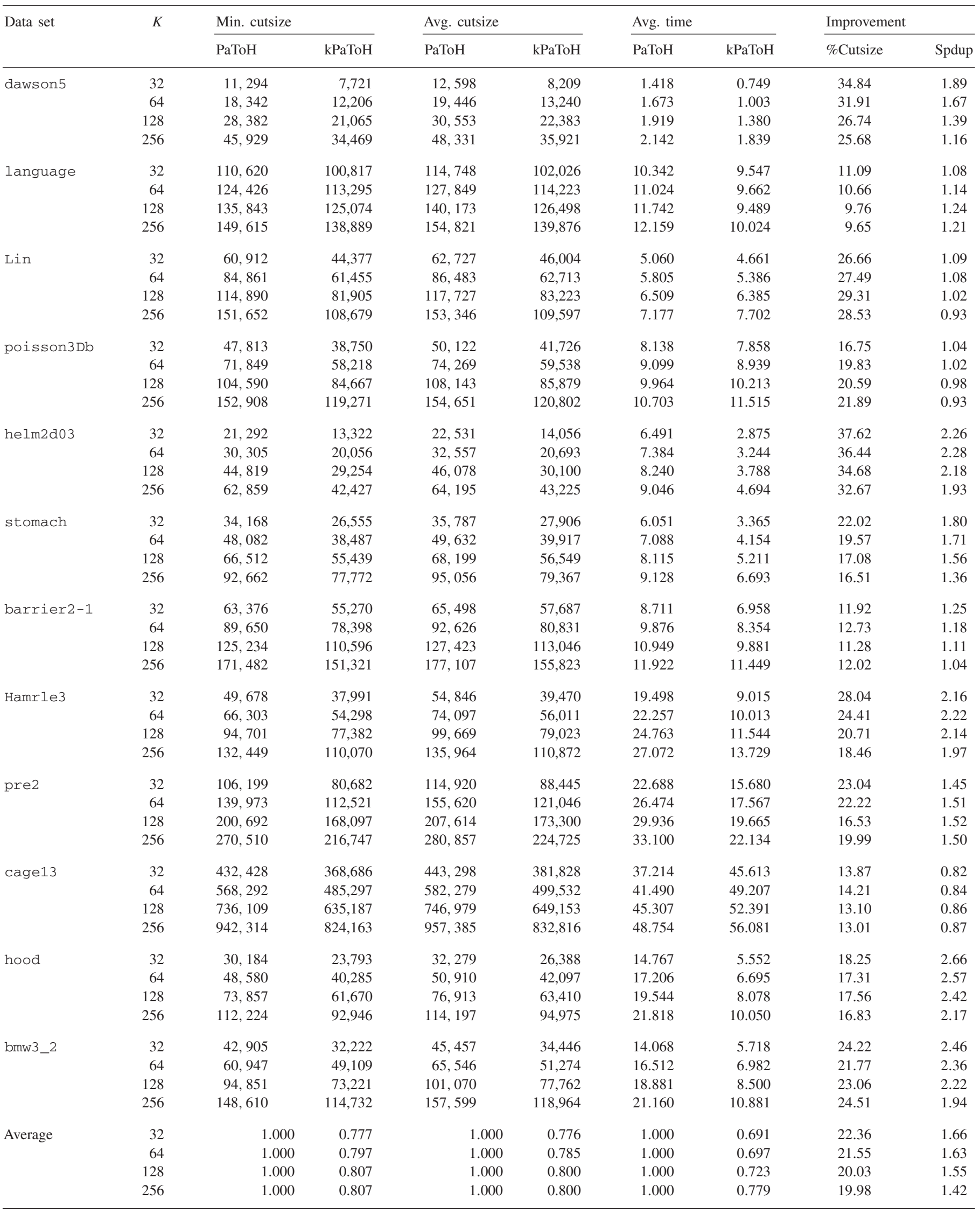


Table 6

Performance of $\mathrm{PaToH}$ and $\mathrm{kPaToH}$ in partitioning hypergraphs with four partitioning constraints

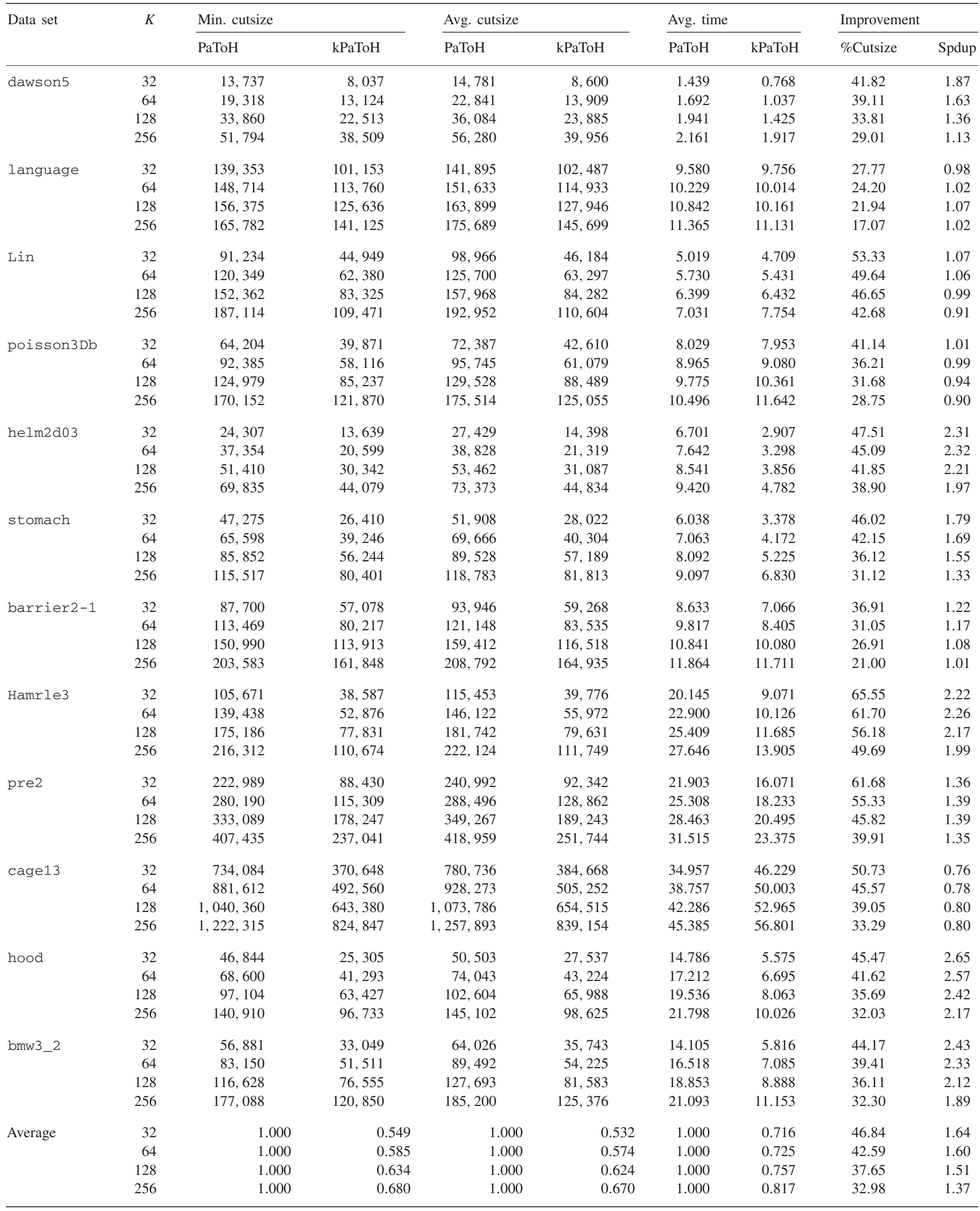


Table 7

Properties of the hypergraphs used in the experiments on partitioning hypergraphs with fixed vertices

\begin{tabular}{|c|c|c|c|c|}
\hline Data set & \# of vertices & \# of nets & $\#$ of pins & Avg. net size \\
\hline $\mathrm{BF}-32$ & 28,930 & 4,800 & 688,018 & 143.34 \\
\hline$B F-64$ & 28,962 & 9,600 & 930,412 & 96.92 \\
\hline $\mathrm{BF}-128$ & 29,026 & 19,200 & $1,335,049$ & 69.53 \\
\hline $\mathrm{CC}-32$ & 56,374 & 4,800 & $1,133,858$ & 236.22 \\
\hline$C C-64$ & 56,406 & 9,600 & $1,472,295$ & 153.36 \\
\hline $\mathrm{CC}-128$ & 56,470 & 19,200 & $2,094,107$ & 109.07 \\
\hline OP- 32 & 68,190 & 4,800 & $1,276,595$ & 265.96 \\
\hline$O P-64$ & 68,222 & 9,600 & $1,629,169$ & 169.70 \\
\hline OP-128 & 68,286 & 19,200 & $1,924,807$ & 100.25 \\
\hline
\end{tabular}

Table 8

Performance of $\mathrm{PaToH}$ and $\mathrm{kPaToH}$ in partitioning hypergraphs with fixed vertices

\begin{tabular}{|c|c|c|c|c|c|c|c|c|c|}
\hline \multirow[t]{2}{*}{ Data set } & \multirow[t]{2}{*}{$K$} & \multicolumn{2}{|c|}{ Min. cutsize } & \multicolumn{2}{|c|}{ Avg. cutsize } & \multicolumn{2}{|c|}{ Avg. time } & \multicolumn{2}{|c|}{ Improvement } \\
\hline & & PaToH & $\mathrm{kPaToH}$ & $\mathrm{PaToH}$ & kPaToH & РaToH & $\mathrm{kPaToH}$ & $\%$ Cutsize & Spdup \\
\hline \multirow[t]{3}{*}{$\mathrm{BF}-32$} & 32 & 9,474 & 7,507 & 9,639 & 7,604 & 5.394 & 2.018 & 21.11 & 2.67 \\
\hline & 64 & 11,343 & 9,379 & 11,799 & 9,623 & 5.906 & 2.186 & 18.44 & 2.70 \\
\hline & 128 & 14,962 & 12,695 & 15,212 & 12,916 & 6.309 & 2.373 & 15.09 & 2.66 \\
\hline \multirow[t]{3}{*}{$B F-64$} & 32 & 17,790 & 13,538 & 18,625 & 13,691 & 5.152 & 2.088 & 26.49 & 2.47 \\
\hline & 64 & 21,473 & 16,583 & 22,010 & 16,867 & 5.726 & 2.309 & 23.37 & 2.48 \\
\hline & 128 & 25,548 & 21,354 & 26,406 & 21,652 & 6.284 & 2.585 & 18.00 & 2.43 \\
\hline \multirow[t]{3}{*}{$\mathrm{BF}-128$} & 32 & 34,522 & 24,234 & 35,751 & 24,568 & 5.770 & 2.709 & 31.28 & 2.13 \\
\hline & 64 & 39,837 & 28,855 & 41,521 & 29,366 & 6.569 & 3.003 & 29.28 & 2.19 \\
\hline & 128 & 47,448 & 36,180 & 48,652 & 36,589 & 7.006 & 3.298 & 24.79 & 2.12 \\
\hline \multirow[t]{3}{*}{$\mathrm{CC}-32$} & 32 & 9,534 & 8,438 & 9,668 & 8,619 & 4.865 & 2.248 & 10.85 & 2.16 \\
\hline & 64 & 12,608 & 10,931 & 12,927 & 11,123 & 5.547 & 2.472 & 13.96 & 2.24 \\
\hline & 128 & 17,635 & 14,796 & 17,873 & 14,956 & 6.172 & 2.771 & 16.32 & 2.23 \\
\hline \multirow[t]{3}{*}{ CC- 64} & 32 & 17,466 & 15,349 & 17,952 & 15,512 & 4.623 & 2.671 & 13.59 & 1.73 \\
\hline & 64 & 21,397 & 19,161 & 21,740 & 19,316 & 5.344 & 3.040 & 11.15 & 1.76 \\
\hline & 128 & 28,088 & 24,685 & 28,729 & 25,010 & 6.012 & 3.282 & 12.94 & 1.83 \\
\hline \multirow[t]{3}{*}{$\mathrm{CC}-128$} & 32 & 33,201 & 28,803 & 34,298 & 28,986 & 5.407 & 3.677 & 15.49 & 1.47 \\
\hline & 64 & 40,036 & 34,947 & 40,677 & 35,225 & 6.233 & 4.218 & 13.40 & 1.48 \\
\hline & 128 & 49,454 & 43,960 & 50,315 & 44,321 & 6.965 & 4.556 & 11.91 & 1.53 \\
\hline \multirow[t]{3}{*}{$O P-32$} & 32 & 8,717 & 6,953 & 8,935 & 7,042 & 18.714 & 6.159 & 21.18 & 3.04 \\
\hline & 64 & 10,367 & 8,530 & 10,804 & 8,622 & 19.485 & 6.372 & 20.19 & 3.06 \\
\hline & 128 & 13,155 & 11,135 & 13,463 & 11,313 & 21.275 & 6.697 & 15.97 & 3.18 \\
\hline \multirow[t]{3}{*}{$O P-64$} & 32 & 15,693 & 12,507 & 16,402 & 12,668 & 17.462 & 6.010 & 22.76 & 2.91 \\
\hline & 64 & 18,823 & 15,028 & 19,399 & 15,178 & 19.317 & 6.291 & 21.76 & 3.07 \\
\hline & 128 & 22,972 & 18,745 & 23,404 & 19,238 & 20.020 & 6.757 & 17.80 & 2.96 \\
\hline \multirow[t]{3}{*}{ OP-128 } & 32 & 30,418 & 22,440 & 31,076 & 22,717 & 13.119 & 5.073 & 26.90 & 2.59 \\
\hline & 64 & 34,735 & 25,876 & 35,157 & 26,505 & 14.981 & 5.425 & 24.61 & 2.76 \\
\hline & 128 & 39,643 & 31,807 & 40,642 & 32,133 & 16.047 & 5.971 & 20.94 & 2.69 \\
\hline \multirow[t]{3}{*}{ Average } & 32 & 1.000 & 0.802 & 1.000 & 0.789 & 1.000 & 0.448 & 21.07 & 2.35 \\
\hline & 64 & 1.000 & 0.814 & 1.000 & 0.804 & 1.000 & 0.437 & 19.57 & 2.42 \\
\hline & 128 & 1.000 & 0.835 & 1.000 & 0.829 & 1.000 & 0.437 & 17.09 & 2.40 \\
\hline
\end{tabular}

In Table 9, we report performance results in terms of the cutsize on a subset of the hypergraphs selected from Table 1 . In each hypergraph, we randomly fix $F=128,256,512$ vertices to $K=128,256,512$ parts in a round-robin fashion and partition the hypergraph using both $\mathrm{PaToH}$ and $\mathrm{kPaToH}$. For $\mathrm{kPaToH}$, we explore the percent improvement due to bipartite graph matching (BGM) and hence try it both with and without BGM. In Table 9, "kPaToH w/ BGM" corresponds to an implementation 
Table 9

Performance of $\mathrm{kPaToH}$ with and without bipartite graph matching (BGM) in partitioning hypergraphs with a randomly selected number $(F)$ of fixed vertices

\begin{tabular}{|c|c|c|c|c|c|c|c|c|}
\hline \multirow[t]{2}{*}{ Data set } & \multirow[t]{2}{*}{$F$} & \multirow[t]{2}{*}{$K$} & \multicolumn{3}{|c|}{ Avg. cutsize } & \multicolumn{2}{|c|}{ \%Improvement } & \multirow{2}{*}{$\begin{array}{l}\% \text { Share of BGM in } \\
\text { the improvement }\end{array}$} \\
\hline & & & $\mathrm{PaToH}$ & $\begin{array}{r}\text { kPaToH } \\
\text { w/o BGM }\end{array}$ & $\begin{array}{r}\text { kPaToH } \\
\text { w/ BGM }\end{array}$ & $\begin{array}{l}\text { kPaToH } \\
\text { w/o BGM }\end{array}$ & $\begin{array}{l}\text { kPaToH } \\
\text { w/ BGM }\end{array}$ & \\
\hline \multirow[t]{9}{*}{ dawson5 } & \multirow[t]{3}{*}{256} & 128 & 26,227 & 23,010 & 20,992 & 12.26 & 19.96 & 38.56 \\
\hline & & 256 & 36,828 & 33,651 & 30,285 & 8.63 & 17.77 & 51.44 \\
\hline & & 512 & 56,948 & 49,299 & 45,409 & 13.43 & 20.26 & 33.71 \\
\hline & \multirow[t]{3}{*}{512} & 128 & 33,075 & 28,005 & 25,345 & 15.33 & 23.37 & 34.42 \\
\hline & & 256 & 43,058 & 38,715 & 34,038 & 10.09 & 20.95 & 51.85 \\
\hline & & 512 & 62,390 & 54,470 & 47,475 & 12.69 & 23.91 & 46.90 \\
\hline & \multirow[t]{3}{*}{1024} & 128 & 46,633 & 37,819 & 34,654 & 18.90 & 25.69 & 26.42 \\
\hline & & 256 & 56,410 & 48,648 & 43,244 & 13.76 & 23.34 & 41.04 \\
\hline & & 512 & 73,354 & 64,288 & 55,273 & 12.36 & 24.65 & 49.86 \\
\hline \multirow[t]{9}{*}{ Lin } & \multirow[t]{3}{*}{256} & 128 & 91,505 & 82,284 & 81,888 & 10.08 & 10.51 & 4.12 \\
\hline & & 256 & 118,981 & 107,683 & 106,572 & 9.50 & 10.43 & 8.95 \\
\hline & & 512 & 155,004 & 140,019 & 138,752 & 9.67 & 10.48 & 7.79 \\
\hline & \multirow[t]{3}{*}{512} & 128 & 94,202 & 84,155 & 83,421 & 10.67 & 11.44 & 6.80 \\
\hline & & 256 & 120,629 & 109,462 & 108,008 & 9.26 & 10.46 & 11.53 \\
\hline & & 512 & 155,286 & 141,911 & 139,816 & 8.61 & 9.96 & 13.55 \\
\hline & \multirow[t]{3}{*}{1024} & 128 & 99,117 & 87,418 & 86,626 & 11.80 & 12.60 & 6.34 \\
\hline & & 256 & 124,302 & 112,920 & 111,368 & 9.16 & 10.41 & 12.00 \\
\hline & & 512 & 158,567 & 145,222 & 142,332 & 8.42 & 10.24 & 17.80 \\
\hline \multirow[t]{9}{*}{ poisson3Db } & \multirow[t]{3}{*}{256} & 128 & 96,136 & 89,682 & 86,009 & 6.71 & 10.53 & 36.27 \\
\hline & & 256 & 132,245 & 123,777 & 118,546 & 6.40 & 10.36 & 38.19 \\
\hline & & 512 & 184,110 & 168,285 & 162,525 & 8.60 & 11.72 & 26.69 \\
\hline & \multirow[t]{3}{*}{512} & 128 & 104,717 & 96,503 & 92,188 & 7.84 & 11.96 & 34.44 \\
\hline & & 256 & 140,800 & 130,267 & 124,592 & 7.48 & 11.51 & 35.02 \\
\hline & & 512 & 191,542 & 174,627 & 164,621 & 8.83 & 14.05 & 37.17 \\
\hline & 1024 & 128 & 126,271 & 109,786 & 104,960 & 13.06 & 16.88 & 22.65 \\
\hline & & 256 & 158,922 & 144,133 & 135,716 & 9.31 & 14.60 & 36.27 \\
\hline & & 512 & 208,279 & 188,168 & 175,242 & 9.66 & 15.86 & 39.13 \\
\hline barrier2-1 & 256 & 128 & 115,348 & 108,922 & 105,160 & 5.57 & 8.83 & 36.93 \\
\hline & & 256 & 154,860 & 144,915 & 139,278 & 6.42 & 10.06 & 36.18 \\
\hline & & 512 & 212,822 & 194,371 & 187,355 & 8.67 & 11.97 & 27.55 \\
\hline & 512 & 128 & 126,627 & 116,548 & 112,418 & 7.96 & 11.22 & 29.07 \\
\hline & & 256 & 166,085 & 153,156 & 145,217 & 7.78 & 12.56 & 38.04 \\
\hline & & 512 & 221,905 & 202,422 & 189,665 & 8.78 & 14.53 & 39.57 \\
\hline & 1024 & 128 & 151,989 & 133,302 & 127,497 & 12.29 & 16.11 & 23.70 \\
\hline & & 256 & 188,254 & 169,670 & 159,889 & 9.87 & 15.07 & 34.48 \\
\hline & & 512 & 243,276 & 218,533 & 202,652 & 10.17 & 16.70 & 39.09 \\
\hline hood & 256 & 128 & 84,003 & 70,391 & 64,793 & 16.20 & 22.87 & 29.14 \\
\hline & & 256 & 112,839 & 99,714 & 90,857 & 11.63 & 19.48 & 40.29 \\
\hline & & 512 & 159,561 & 142,146 & 131,641 & 10.91 & 17.50 & 37.62 \\
\hline & 512 & 128 & 107,231 & 82,145 & 75,616 & 23.39 & 29.48 & 20.65 \\
\hline & & 256 & 133,568 & 111,861 & 100,191 & 16.25 & 24.99 & 34.96 \\
\hline & & 512 & 175,277 & 154,121 & 136,864 & 12.07 & 21.92 & 44.93 \\
\hline & 1024 & 128 & 149,171 & 106,871 & 99,501 & 28.36 & 33.30 & 14.84 \\
\hline & & 256 & 172,577 & 136,753 & 122,510 & 20.76 & 29.01 & 28.45 \\
\hline & & 512 & 212,997 & 179,269 & 156,507 & 15.83 & 26.52 & 40.29 \\
\hline bomw3_2 & 256 & 128 & 89,754 & 78,213 & 72,883 & 12.86 & 18.80 & 31.59 \\
\hline & & 256 & 121,386 & 112,222 & 103,552 & 7.55 & 14.69 & 48.61 \\
\hline & & 512 & 174,406 & 161,878 & 152,451 & 7.18 & 12.59 & 42.94 \\
\hline & 512 & 128 & 110,871 & 90,326 & 83,413 & 18.53 & 24.77 & 25.18 \\
\hline & & 256 & 141,551 & 125,157 & 113,760 & 11.58 & 19.63 & 41.01 \\
\hline & & 512 & 189,793 & 174,442 & 156,349 & 8.09 & 17.62 & 54.10 \\
\hline & 1024 & 128 & 150,756 & 115,426 & 107,445 & 23.43 & 28.73 & 18.43 \\
\hline & & 256 & 178,616 & 149,789 & 136,220 & 16.14 & 23.74 & 32.01 \\
\hline & & 512 & 223,685 & 200,207 & 176,784 & 10.50 & 20.97 & 49.94 \\
\hline
\end{tabular}


Table 10

Averages of the results provided in Table 9 over $F$ and $K$

\begin{tabular}{|c|c|c|c|c|c|c|c|}
\hline \multicolumn{4}{|c|}{ Averages over $F$} & \multicolumn{4}{|c|}{ Averages over $K$} \\
\hline \multirow[t]{2}{*}{$F$} & \multicolumn{2}{|c|}{$\%$ Improvement } & \multirow[t]{2}{*}{$\%$ Share of BGM in the improvement } & \multirow[t]{2}{*}{$K$} & \multicolumn{2}{|c|}{$\%$ Improvement } & \multirow[t]{2}{*}{$\%$ Share of BGM in the improvement } \\
\hline & $\begin{array}{l}\text { kPaToH } \\
\text { w/o BGM }\end{array}$ & $\begin{array}{l}\text { kPaToH } \\
\text { w/ BGM }\end{array}$ & & & $\begin{array}{l}\text { kPaToH } \\
\text { w/o BGM }\end{array}$ & $\begin{array}{l}\text { kPaToH } \\
\text { w/ BGM }\end{array}$ & \\
\hline 256 & 9.57 & 14.38 & 32.03 & 128 & 14.18 & 18.73 & 24.42 \\
\hline 512 & 11.40 & 17.46 & 33.29 & 256 & 10.64 & 16.61 & 34.46 \\
\hline 1024 & 14.10 & 20.24 & 29.60 & 512 & 10.25 & 16.75 & 36.03 \\
\hline
\end{tabular}

of the approach described in Section 4, whereas " $\mathrm{kPaToH}$ w/o BGM" corresponds to an implementation in which the fixed vertex sets are arbitrarily matched with the ordinary vertex parts.

Basically, $\mathrm{kPaToH}$ provides superior results over $\mathrm{PaToH}$ in partitioning hypergraphs with fixed vertices due to (i) $K$-way refinement and (ii) BGM performed during the the initial partitioning phase. We provide Table 9 to illustrate the share of these two factors in the overall cutsize improvement. According to Table 9, the share of BGM in the total cutsize improvement is quite variable, ranging between $4.12 \%$ and $54.10 \%$. In general, better results are achieved by BGM for hypergraphs having large net sizes. It is hard to make a judgment about the behavior with increasing $F$ and $K$ values. This is due to the fact that many other factors affect the performance of BGM. Among these factors, the performance is dependent on the connectivity of the regular and fixed vertices, distribution of fixed vertices on the parts, the ratio of $F$ to the number of regular vertices, and the ratio of $F$ to $K$. These are hard to assess (also see [1] for comments on the bipartitioning case). The averages over $F$ and $K$ values are provided in Table 10 .

\section{Conclusion}

We argued that the hypergraph partitioning with multiple constraints and fixed vertices problems should be tackled with a direct $K$-way refinement approach. In order to support our claim, we presented a careful implementation of a multi-level direct $K$-way refinement algorithm. We discussed extensions of this algorithm for partitioning hypergraphs with multiple constraints and fixed vertices. Extension to the multi-constraint case adopts standard hypergraph partitioning techniques. In order to extend the algorithm to the fixed vertex case, we proposed specialized coarsening and initial partitioning with a novel formulation that uses bipartite graph matching. The experiments conducted on benchmark data sets indicate that the proposed algorithm is quite fast and effective in minimizing the cutsize compared to the state-of-the-art hypergraph partitioning tool $\mathrm{PaToH}$. Especially, in the multi-constraint and fixed vertices domain, the obtained results are quite promising in terms of both execution time and solution quality. On average, the cutsize improvement of $\mathrm{kPaToH}$ over $\mathrm{PaToH}$ is around $20 \%$ and $40 \%$ at the 2-constraint and 4-constraint cases, respectively. Similarly, in partitioning hypergraphs with fixed vertices, $\mathrm{kPa}-$ ToH outperforms PaToH by up to $33 \%$ in cutsize as it runs 2.3 times faster on average.

\section{References}

[1] C.J. Alpert, A.E. Caldwell, A.B. Kahng, I.L. Markov, Hypergraph partitioning with fixed vertices, IEEE Trans. Comput.-Aided Design 19 (2) (2000) 267-272.

[2] C.J. Alpert, A.B. Kahng, Recent directions in netlist partitioning: a survey, VLSI J. 19 (1-2) (1995) 1-81.

[3] C. Ashcraft, Compressed graphs and the minimum degree algorithm, SIAM J. Sci. Comput. 16 (6) (1995) 1404-1411.

[4] C. Aykanat, A. Pinar, Ü.V. Çatalyürek, Permuting sparse rectangular matrices into block-diagonal form, SIAM J. Sci. Comput. 25 (6) (2004) 1860-1879.

[5] C. Berge, Graphs and Hypergraphs, North-Holland Publishing Company, Amsterdam, 1973.

[6] R.H. Bisseling, J. Byrka, S. Cerav-Erbas, N. Gvozdenovic, M. Lorenz, R. Pendavingh, C. Reeves, M. Roger, A. Verhoeven, Partitioning a call graph, in: Second International Workshop on Combinatorial Scientific Computing, 2005.

[7] R.H. Bisseling, I. Flesch, Mondriaan sparse matrix partitioning for attacking cryptosystems by a parallel block Lanczos algorithm: a case study, Parallel Comput. 32 (7) (2006) 551-567.

[8] T.N. Bui, C. Jones, A heuristic for reducing fill in sparse matrix factorization, in: Proceedings of the Sixth SIAM Conference on Parallel Processing for Scientific Computing, 1993, pp. 445-452.

[9] A. Caldwell, A. Kahng, I. Markov, Improved algorithms for hypergraph bipartitioning, in: Proceedings of the IEEE ACM Asia and South Pacific Design Automation Conference, 2000, pp. 661-666.

[10] B.B. Cambazoglu, C. Aykanat, Hypergraph-partitioning-based remapping models for image-space-parallel direct volume rendering of unstructured grids, IEEE Trans. Parallel Distributed Systems 18 (1) (2007) 3-16.

[11] C. Chang, T.M. Kurc, A. Sussman, Ü.V. Çatalyürek, J.H. Saltz, A hypergraph-based workload partitioning strategy for parallel data aggregation, in: SIAM Conference on Parallel Processing for Scientific Computing, 2001.

[12] G. Chartrand, O.R. Oellermann, Applied and Algorithmic Graph Theory, McGraw-Hill, New York, 1993.

[13] C. Clifton, R. Cooley, J. Rennie, TopCat: data mining for topic identification in a text corpus, IEEE Trans. Knowledge Data Eng. 16 (8) (2004) 949-964.

[14] Ü.V. Çatalyürek, ISPD98 benchmark 〈http://bmi.osu.edu/ umit/PaToH/ ispd98.html .

[15] Ü.V. Çatalyürek, C. Aykanat, Decomposing irregularly sparse matrices for parallel matrix-vector multiplication, Lecture Notes in Computer Science, vol. 1117, 1996, pp. 75-86.

[16] Ü.V. Çatalyürek, C. Aykanat, PaToH: partitioning tool for hypergraphs, Technical Report, Department of Computer Engineering, Bilkent University, 1999.

[17] Ü.V. Çatalyürek, C. Aykanat, Hypergraph-partitioning-based decomposition for parallel sparse-matrix vector multiplication, IEEE Trans. Parallel Distributed Systems 10 (7) (1999) 673-693.

[18] Ü.V. Çatalyürek, C. Aykanat, A fine-grain hypergraph model for 2D decomposition of sparse matrices, in: Proceedings of the 15th International Parallel and Distributed Processing Symposium, 2001, p. 118. 
[19] Ü.V. Çatalyürek, C. Aykanat, A hypergraph-partitioning approach for coarse-grain decomposition, in: Proceedings of the 2001 ACM/IEEE Conference on Supercomputing, 2001, p. 28.

[20] A. Dasdan, C. Aykanat, Two novel multiway circuit partitioning algorithms using relaxed locking, IEEE Trans. Comput.-Aided Design Integrated Circuits Systems 16 (2) (1997) 169-178.

[21] T. Davis, University of Florida Sparse Matrix Collection 〈http://www. cise.ufl.edu/research/sparse/matrices〉, NA Digest 97 (23) (June 7, 1997).

[22] E. Demir, C. Aykanat, B.B. Cambazoglu, Clustering spatial networks for aggregate query processing: a hypergraph approach, Inform. Systems, in press.

[23] E. Demir, C. Aykanat, B.B. Cambazoglu, A link-based storage scheme for efficient aggregate query processing on clustered road networks, Technical Report, BU-CE-0707, Department of Computer Engineering, Bilkent University, 2007.

[24] K.D. Devine, E.G. Boman, R.T. Heaphy, R. Bisseling, Ü.V. Çatalyürek, Parallel hypergraph partitioning for scientific computing, in: Proceedings of the IEEE International Parallel and Distributed Processing Symposium, 2006.

[25] K.D. Devine, E.G. Boman, R.T. Heaphy, B. Hendrickson, C. Vaughan, Zoltan data management services for parallel dynamic applications, Comput. Sci. Eng. 4 (2) (2002) 90-97.

[26] N.J. Dingle, P.G. Harrison, W.J. Knottenbelt, Uniformization and hypergraph partitioning for the distributed computation of response time densities in very large Markov models, J. Parallel Distributed Comput. 64 (8) (2004) 908-920.

[27] I.S. Duff, S. Riyavong, M.B. van Gijzen, Parallel preconditioners based on partitioning sparse matrices, Technical Report, TR/PA/04/114, CERFACS, 2004

[28] C.M. Fiduccia, R.M. Mattheyses, A linear-time heuristic for improving network partitions, in: Proceedings of the 19th ACM/IEEE Design Automation Conference, 1982, pp. 175-181.

[29] M.K. Goldberg, M. Burnstein, Heuristic improvement technique for bisection of VLSI networks, in: Proceedings of the IEEE International Conference on Computer Design, 1983, pp. 122-125.

[30] B. Hendrickson, R. Leland, The Chaco user's guide: version 2.0, Technical Report, SAND94-2692, Sandia National Laboratories, 1994.

[31] B. Hendrickson, E. Rothberg, Improving the run time and quality of nested dissection ordering, SIAM J. Sci. Comput. 20 (2) (1998) 468-489.

[32] G. Karypis, R. Aggarwal, V. Kumar, S. Shekhar, Multilevel hypergraph partitioning: applications in VLSI domain, IEEE Trans. Very Large Scale Integration Systems 7 (1) (1999) 69-79.

[33] G. Karypis, V. Kumar, hMETIS: a hypergraph partitioning package, Technical Report, Department of Computer Science, University of Minnesota, 1998.

[34] G. Karypis, V. Kumar, MeTiS: a software package for partitioning unstructured graphs, partitioning meshes and computing fill-reducing orderings of sparse matrices, Technical Report, Department of Computer Science, University of Minnesota, 1998.

[35] G. Karypis, V. Kumar, Multilevel algorithms for multi-constraint graph par-titioning, in: Proceedings of the 1998 ACM/IEEE Conference on Supercomputing, 1998, pp. 1-13.

[36] G. Karypis, V. Kumar, Multilevel $k$-way hypergraph partitioning, VLSI Design 11 (3) (2000) 285-300.

[37] K. Kaya, C. Aykanat, Iterative-improvement-based heuristics for adaptive scheduling of tasks sharing files on heterogeneous master-slave environments, IEEE Trans. Parallel Distributed Systems 17 (8) (2006) 883-896.

[38] K. Kaya, B. Ucar, C. Aykanat, Heuristics for scheduling file-sharing tasks on heterogeneous systems with distributed repositories, J. Parallel Distributed Comput. 67 (3) (2007) 271-285.

[39] B.W. Kernighan, S. Lin, An efficient heuristic procedure for partitioning graphs, Bell System Technical J. 49 (1970) 291-307.
[40] G. Khanna, N. Vydyanathan, T.M. Kurc, Ü.V. Çatalyürek, P. Wyckoff, J. Saltz, P. Sadayappan, A hypergraph partitioning based approach for scheduling of tasks with batch-shared IO, in: Proceedings of Cluster Computing and Grid, 2005.

[41] M. Koyuturk, C. Aykanat, Iterative-improvement-based declustering heuristics for multi-disk databases, Inform. Systems 30 (1) (2005) 47-70.

[42] T. Lengauer, Combinatorial Algorithms for Integrated Circuit Layout, Wiley-Teubner, Chichester, 1990.

[43] D.R. Liu, M.Y. Wu, A hypergraph based approach to declustering problems, Distributed Parallel Databases 10 (3) (2001) 269-288.

[44] M.M. Ozdal, C. Aykanat, Hypergraph models and algorithms for datapattern-based clustering, Data Mining Knowledge Discovery 9 (1) (2004) $29-57$.

[45] D.G. Schweikert, B.W. Kernighan, A proper model for the partitioning of electrical circuits, in: Proceedings of the 9th Workshop on Design Automation, 1972, pp. 57-62.

[46] S. Shekhar, C.-T. Lu, S. Chawla, S. Ravada, Efficient join-index-based spatial-join processing: a clustering approach, IEEE Trans. Knowledge Data Eng. 14 (6) (2002) 1400-1421.

[47] H.D. Simon, S.-H. Teng, How good is recursive bisection?, SIAM J. Sci. Comput. 18 (5) (1997) 1436-1445.

[48] A. Trifunovic, W.J. Knottenbelt, Parkway2.0: a parallel multilevel hypergraph partitioning tool, in: Proceedings of the International Symposium on Computer and Information Sciences, 2004, pp. 789-800.

[49] B. Uçar, C. Aykanat, Encapsulating multiple communication-cost metrics in partitioning sparse rectangular matrices for parallel matrix-vector multiplies, SIAM J. Sci. Comput. 25 (6) (2004) 1837-1859.

[50] B. Uçar, C. Aykanat, Revisiting hypergraph models for sparse matrix partitioning, SIAM Rev. 49 (4) (2007) 543-732.

[51] B. Uçar, C. Aykanat, Partitioning sparse matrices for parallel preconditioned iterative methods, SIAM J. Sci. Comput. 29 (4) (2007) 1683-1709

[52] B. Uçar, C. Aykanat, M.C. Pınar, T. Malas, Parallel image restoration using surrogate constraints methods, J. Parallel Distributed Comput. 67 (2) (2007) 186-204.

[53] B. Vastenhouw, R.H. Bisseling, A two-dimensional data distribution method for parallel sparse matrix-vector multiplication, SIAM Rev. 47 (1) (2005) 67-95.

[54] C. Walshaw, M. Cross, K. McManus, Multiphase mesh partitioning, Appl. Math. Modelling 25 (2000) 123-140.

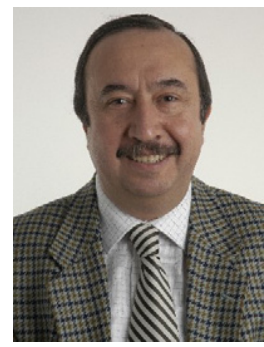

Cevdet Aykanat received the BS and MS degrees from Middle East Technical University, Ankara, Turkey, both in electrical engineering, and the $\mathrm{PhD}$ degree from Ohio State University, Columbus, in electrical and computer engineering. He was a Fulbright scholar during his $\mathrm{PhD}$ studies. He worked at the Intel Supercomputer Systems Division, Beaverton, Oregon, as a research associate. Since 1989, he has been affiliated with the Department of Computer Engineering, Bilkent University, Ankara, Turkey, where he is currently a professor. His research interests mainly include parallel computing, parallel scientific computing and its combinatorial aspects, parallel computer graphics applications, parallel data mining, graph and hypergraph partitioning, load balancing, neural network algorithms, high performance information retrieval systems, parallel and distributed web crawling, parallel and distributed databases, and grid computing. He has (co)authored over 40 technical papers published in academic journals indexed in SCI. He is the recipient of the 1995 Young Investigator Award of The Scientific and Technological Research Council of Turkey. He is a member of the ACM and the IEEE Computer Society. He has been recently appointed as a member of IFIP Working Group 10.3 (Concurrent Systems). 


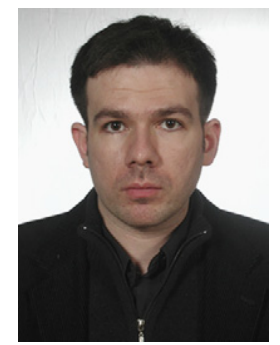

Berkant Barla Cambazoglu received his BS, $\mathrm{MS}$, and $\mathrm{PhD}$ degrees all in computer engineering from the Computer Engineering Department of Bilkent University in 1997, 2000, and 2006, respectively. He has worked in several research projects funded by the Scientific and Technological Research Council of Turkey, the European Union Sixth Framework Program, and the National Cancer Institute. He is currently working as a postdoctoral researcher at the Biomedical Informatics Department of the Ohio State University. His research interests include high

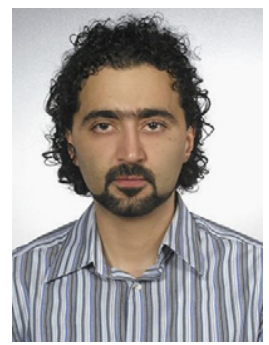

Bora Ucar received the $\mathrm{PhD}$ degree (2005) in computer engineering from Bilkent University, Ankara, Turkey. He is currently working at the Parallel Algorithms Project, CERFACS, France. His research interests are combinatorial scientific computing, parallel and high performance computing, and sparse matrix computations.

performance computing, scientific visualization, information retrieval, data mining, and grid middleware. 